NIST Special Publication 800-156

\title{
Representation of \\ PIV Chain-of-Trust for Import and Export
}

This publication is available free of charge from: http://dx.doi.org/10.6028/NIST.SP.800-156

\section{O M P U TER SEC UR I T Y}

\author{
Hildegard Ferraiolo \\ Ramaswamy Chandramouli \\ Ketan Mehta \\ Jason Mohler \\ Stephen Skordinski \\ Steven Brady
}

COMPUTER SECUR I T Y


NIST Special Publication 800-156

\title{
Representation of PIV Chain-of-Trust for Import and Export
}

\author{
Hildegard Ferraiolo \\ Ramaswamy Chandramouli \\ Ketan Mehta \\ Computer Security Division \\ Information Technology Laboratory \\ Jason Mohler \\ Stephen Skordinski \\ Steven Brady \\ Electrosoft Services Inc. \\ Reston, VA
}

This publication is available free of charge from: http://dx.doi.org/10.6028/NIST.SP.800-156

May 2016

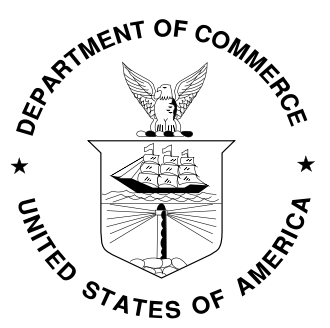

U.S. Department of Commerce

Penny Pritzker, Secretary 


\title{
Authority
}

This publication has been developed by NIST to further its statutory responsibilities under the Federal Information Security Modernization Act (FISMA) of 2014, 44 U.S.C. § 3541 et seq., Public Law 113283. NIST is responsible for developing information security standards and guidelines, including minimum requirements for federal information systems, but such standards and guidelines shall not apply to national security systems without the express approval of appropriate federal officials exercising policy authority over such systems. This guideline is consistent with the requirements of the Office of Management and Budget (OMB) Circular A-130.

Nothing in this publication should be taken to contradict the standards and guidelines made mandatory and binding on federal agencies by the Secretary of Commerce under statutory authority. Nor should these guidelines be interpreted as altering or superseding the existing authorities of the Secretary of Commerce, Director of the OMB, or any other federal official. This publication may be used by nongovernmental organizations on a voluntary basis and is not subject to copyright in the United States. Attribution would, however, be appreciated by NIST.

\section{National Institute of Standards and Technology Special Publication 800-156 Natl. Inst. Stand. Technol. Spec. Publ. 800-156, 48pages (May 2016) CODEN: NSPUE2}

This publication is available free of charge from: http://dx.doi.org/10.6028/NIST.SP.800-156

Certain commercial entities, equipment, or materials may be identified in this document in order to describe an experimental procedure or concept adequately. Such identification is not intended to imply recommendation or endorsement by NIST, nor is it intended to imply that the entities, materials, or equipment are necessarily the best available for the purpose.

There may be references in this publication to other publications currently under development by NIST in accordance with its assigned statutory responsibilities. The information in this publication, including concepts and methodologies, may be used by federal agencies even before the completion of such companion publications. Thus, until each publication is completed, current requirements, guidelines, and procedures, where they exist, remain operative. For planning and transition purposes, Federal agencies may wish to closely follow the development of these new publications by NIST.

Organizations are encouraged to review all draft publications during public comment periods and provide feedback to NIST. Many NIST cybersecurity publications, other than the ones noted above, are available at http://csrc.nist.gov/publications.

\section{Comments on this publication may be submitted to:}

\author{
National Institute of Standards and Technology \\ Attn: Computer Security Division, Information Technology Laboratory \\ 100 Bureau Drive (Mail Stop 8930) Gaithersburg, MD 20899-8930 \\ Email: piv comments@nist.gov
}

All comments are subject to release under the Freedom of Information Act (FOIA). 


\section{Reports on Computer Systems Technology}

The Information Technology Laboratory (ITL) at the National Institute of Standards and Technology (NIST) promotes the U.S. economy and public welfare by providing technical leadership for the Nation's measurement and standards infrastructure. ITL develops tests, test methods, reference data, proof of concept implementations, and technical analyses to advance the development and productive use of information technology. ITL's responsibilities include the development of management, administrative, technical, and physical standards and guidelines for the cost-effective security and privacy of other than national security-related information in federal information systems. The Special Publication 800-series reports on ITL's research, guidelines, and outreach efforts in information system security, and its collaborative activities with industry, government, and academic organizations.

\section{Abstract}

This document provides a common XML-based data representation of a chain-of-trust record to facilitate the exchange of Personal Identity Verification (PIV) Card enrollment data. The exchanged record is the basis to personalize a PIV Card for a transferred employee and also for service providers to personalize a PIV Card on behave of client federal agencies.

\section{Keywords}

enrollment record; Federal Information Processing Standards (FIPS) 201; HSPD-12; identification; identity infrastructure; Personal Identity Verification (PIV); PIV Architecture; PIV Card; PIV chain-of-trust; XML information sharing

\section{Acknowledgement}

The authors of SP 800-156 gratefully acknowledge and appreciate the support and contributions by many in the public and private sectors whose helpful and beneficial comments greatly enhance the utility of this publication. Special thanks to Stephen Sill and Matthew Arnold from the GSA HSPD12 Managed Service Office (MSO) for their review and contributions to this document. 


\section{Table of Contents}

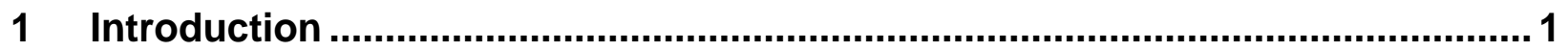

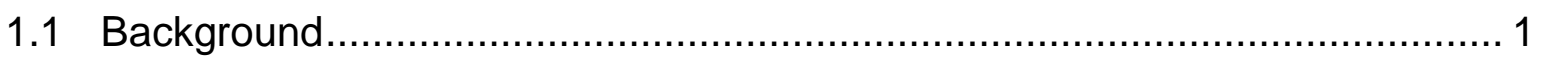

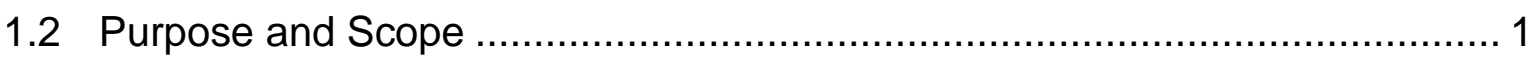

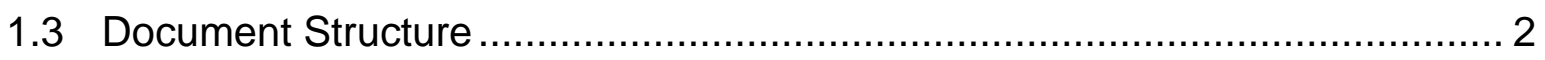

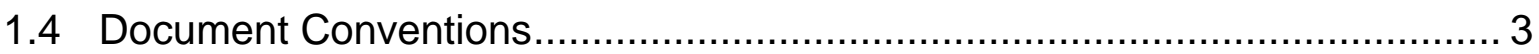

2 Chain-of-Trust Data Requirements and Namespaces.......................................... 4

2.1 Namespaces Declaration for Chain-of-Trust Schema .................................. 4

3 Chain-of-Trust Schema Description ................................................................ 6

3.1 Schema Header and Namespace Declarations ......................................... 6

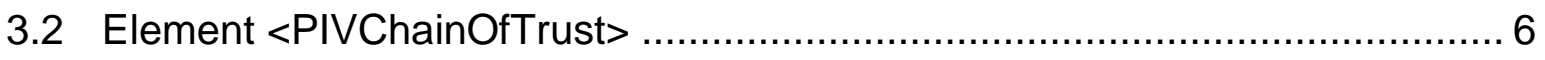

3.3 Complex Type PIVChainOfTrustType ………......................................... 6

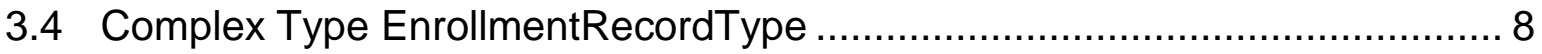

3.5 Complex Type PersonallnformationType ……........................................ 9

3.6 Complex Type AdjudicationInformationType ............................................ 11

3.7 Complex Type EnrollmentPackageType …............................................ 12

3.8 Complex Type EPBiographicType …................................................... 13

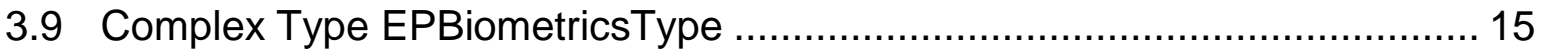

3.10 Complex Type EPDocumentType .......................................................... 16

3.11 Complex Type PIVCardTopographyType ……..................................... 20

4 Chain-of-trust Record Encryption in Transit ................................................. 24

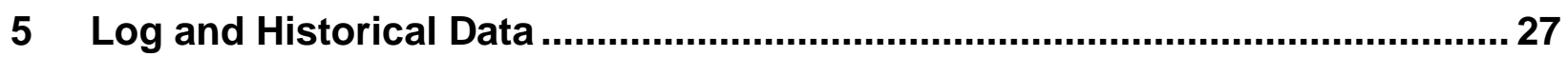

\section{List of Appendices}

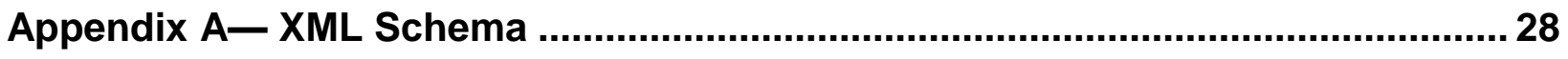

Appendix B- XML Sample PIVChain OfTrust Record.............................................. 34

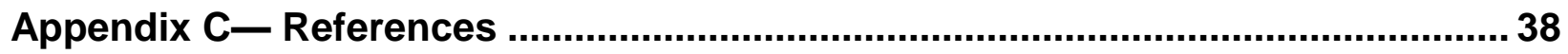

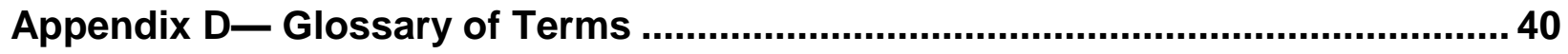

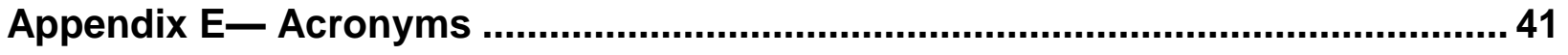




\section{List of Tables}

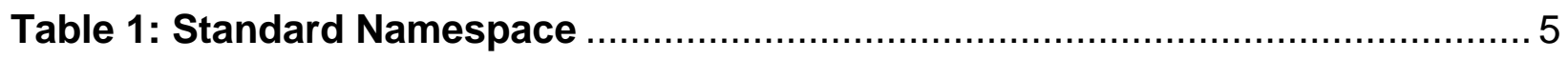

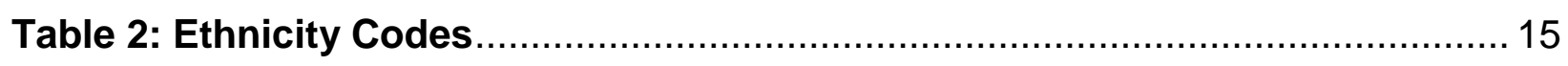




\section{Introduction}

\subsection{Background}

The Homeland Security Presidential Directive 12 [HSPD12] called for a common identification standard to be adopted governing the interoperable use of identity credentials to allow physical and logical access to federal government locations and systems. The Federal Information Processing Standard (FIPS) 201 [FIPS201], "Personal Identity Verification (PIV) of Federal Employees and Contractors," was developed as the standard for the identity credentials hosted on a smart card called the PIV Card. [FIPS201] defines the identity proofing, registration, and issuance requirements for issuing PIV Cards to Federal Government employees and contractors.

Data collected during identity proofing, registration, and issuance may be maintained in a chain-of-trust record. As per FIPS 201-2, "a card issuer may optionally maintain, for each PIV Card issued, a documentary chain-of-trust for the identification data it collects. The chain-of-trust is a sequence of related enrollment data records that are created and maintained through the methods of contemporaneous acquisition of data within each enrollment data record, and biometric matching of samples between enrollment data records."

The chain-of-trust offers process efficiencies because a PIV Card can be re-issued based on the most current chain-of-trust record, and more importantly, can avoid having to repeat the identity proofing and re-registration (re-enrollment) process. Departments and agencies that implement a chain-of-trust will also be able to transfer the record to another agency or to a service provider, so that the receiving agency or service provider can use the record to issue a PIV Card rather than re-enroll an applicant. This Special Publication provides the representation of a chain-of-trust for import and export between PIV Card issuers.

\subsection{Purpose and Scope}

[FIPS201] describes three use cases for a chain-of-trust: 1) to extend enrollment, 2) to reissue a PIV Card, and 3) to transfer PIV Card enrollment records to another federal issuer or to a service provider. The purpose of this document is to provide the data representation of a chain-of-trust record for the transferal use case. The data representation is based on a common XML (Extensible Markup Language) schema to facilitate interoperable information sharing and data exchange. The sending and receiving federal agencies will be able to exchange the chain-of-trust data according to the specifications provided in this document. Similarly, a service provider will be able to receive chain-of-trust records from different client agencies that are based on a common XML schema specified in this document. This document also provides support for data integrity through digital signatures and for confidentiality through encryption of chain-oftrust data, in transit and at rest. 
There are two use cases within the transferal scenario which this document supports:

Agency to agency: In this use case, an existing PIV cardholder from agency A is transferring to agency B, where he/she will require a new PIV Card issued by agency B. Rather than re-enrolling the user, the chain-of-trust record is sent, (upon Agency B's request) from agency $A$ to agency $B$ such that agency $B$ is able to reuse the enrollment data, thus reducing the time and effort required for agency B to issue a new PIV Card.

Agency to service provider: In this use case, an agency does not directly issue PIV Cards to their employees and contractors, but instead utilizes a separate service provider for issuance of PIV Cards. The agency can use the chain-of-trust to send PIV Card enrollment data collected by the agency to the service provider.

It is anticipated that more optional elements will be populated in the chain-of-trust XML record for the agency to service provider use case than the agency to agency use case. In the agency to service provider exchange, the agency is likely to indicate several optional elements that are not applicable in the agency to agency use case. Examples of these elements include those that would be printed on a card and are provided by the agency to the service provider, such as <EmployeeAffiliation>, $<$ AgencyDepartmentOrOrganization>, <AffiliationColorCode>, and <Rank>.

Regardless of the use case, the goal is to provide a common chain-of-trust schema to facilitate information sharing and data exchange between different issuers.

\subsection{Document Structure}

The remainder of this document is divided into the following sections and appendices:

- Section 2, Chain-of-Trust Data Requirements and Namespaces, discusses the representation of chain-of-trust data in XML schemas.

- $\quad$ Section 3, Chain-of-Trust Schema Description, documents the detail of chain-oftrust XML schema.

- $\quad$ Section 4, Chain-of-trust Record Encryption in Transit, identifies the requirements for encrypting chain-of-trust data.

- $\quad$ Section 5, Log and Historical Data, discusses the requirement for the chain-oftrust producer to maintain records related to the cardholder.

- Appendix A contains the XML schema for the chain-of-trust.

- $\quad$ Appendix B contains a sample XML chain-of-trust data record.

- $\quad$ Appendix C identifies references used in this document.

- $\quad$ Appendix D provides a glossary of terms used in this document. 
- Appendix E provides a list of acronyms and abbreviations used in this document.

\subsection{Document Conventions}

Throughout this document key words are used to identify requirements. The key words "optional", "required", "shall", "shall not", and "should" are used. These words are a subset of the IETF Request For Comments (RFC) 2119 [RFC2119] key words, and have been chosen based on convention in other normative documents. In addition to the key words, the words 'need,' 'can,' and 'may' are used in this document, but are not intended to be normative. 


\section{Chain-of-Trust Data Requirements and Namespaces}

[FIPS201] recommends that the following data be included in the chain-of-trust:

- A log of activities that documents who took the action, what action was taken, when and where the action took place, and what identification data was collected.

- An enrollment data record that contains the most recent collection of each of the biometric data collected. The enrollment data record describes the circumstances of biometric acquisition including the name and role of the acquiring agent, the office and organization, time, place, and acquisition method. The enrollment data record may also document unavailable biometric data or failed attempts to collect biometric data. The enrollment data record may contain historical biometric data.

- The most recent unique identifiers (i.e., Federal Agency Smart Credential Number (FASC-N) and Universally Unique Identifier (UUID)) issued to the individual's card. The record may contain historical unique identifiers.

- Information about the authorizing entity who has approved the issuance of a credential.

- Current status of the background investigation, including the results of the investigation once completed.

- The evidence of authorization if the credential is issued under a pseudonym.

- Any data or any subsequent changes in the data about the cardholder. If the changed data is the cardholder's name, then the issuer should include the evidence of a formal name change.

The following sections provide an XML schema representation of the baseline data included in the chain-of-trust.

This schema has been authored to address the broadest envisioned range of chain-of-trust data to be able to transfer PIV Card enrollment records across federal agencies and to a service provider. Users of this XML schema may extend the schema as needed. Extending the XML schema will allow the exchange of additional elements to meet requirements for specialized exchange of chain-of-trust data. However, extending the XML schema will also result in custom exchange that is less interoperable.

\subsection{Namespaces Declaration for Chain-of-Trust Schema}

XML namespace prefixes are used throughout this specification to stand for their respective namespaces as follows: 
Table 1: Standard Namespace

\begin{tabular}{|l|l|l|}
\hline Prefix & XML Namespace & Comments \\
\hline$d s:$ & http://www.w3.org/2000/09/xmldsig\# & $\begin{array}{l}\text { This is the W3C XML Signature } \\
\text { namespace [XMLSig]. }\end{array}$ \\
\hline nist: & $\begin{array}{l}\text { http://csrc.nist.gov/ns/piv/chain- } \\
\text { of-trust/1.0 }\end{array}$ & $\begin{array}{l}\text { This is the NIST's Computer } \\
\text { Security Division's chain-of-trust } \\
\text { namespace. }\end{array}$ \\
\hline$x e n c:$ & http://www.w3.org/2001/04/xmlenc\# & $\begin{array}{l}\text { This is the W3C XML Encryption } \\
\text { namespace [XMLEnc]. }\end{array}$ \\
\hline$x s:$ & http://www.w3.org/2001/XMLSchema & $\begin{array}{l}\text { This is the schema for XML } \\
\text { schemas namespace } \\
\text { [XMLSchema]. }\end{array}$ \\
\hline
\end{tabular}

The chain of trust schema defined in this document can be downloaded at http://csrc.nist.gov/schema/piv/chain-of-trust/piv-chain-of-trust-1.0.xsd.

This specification uses the following typographical conventions in the text: <Element>, Defined Type, XML and XML schema. 


\section{Chain-of-Trust Schema Description}

The chain-of-trust data is encoded in the XML format specified in this document. The chain-of-trust XML records are intended to be used in a direct-exchange. While log and historical data are not included in the XML chain-of-trust record, the issuer of the chainof-trust record should be able to correlate the chain-of-trust record to the associated log and historic data, if requested to do so by the recipient of the chain-of-trust record. The $<$ PIVTrustChain> element may include the records for multiple subjects, with each $<$ EnrollmentRecord $>$ element containing the record for a single subject.

This section contains an overview of the elements that may appear in a chain-of-trust XML file.

\subsection{Schema Header and Namespace Declarations}

The following schema fragment defines the XML namespaces and other header information for the PIV chain-of-trust schema:

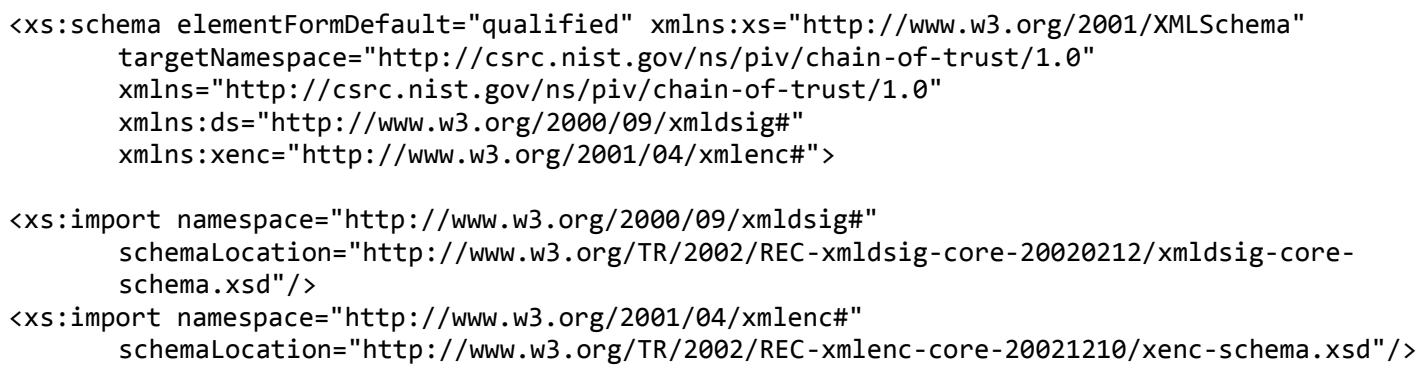

\subsection{Element <PIVChainOfTrust $>$}

The following schema fragment defines the <PIVChainOfTrust> element:

<xs:element name="PIVChainOfTrust" type="PIVChainOfTrustType" />

The <PIVChainOfTrust> element is of PIVChainOfTrust Type complex type, and carries one or more <EnrollmentRecord $>$ elements.

\subsection{Complex Type PIVChainOfTrustType}

The following schema fragment defines the PIVChainOfTrust Type complex type:

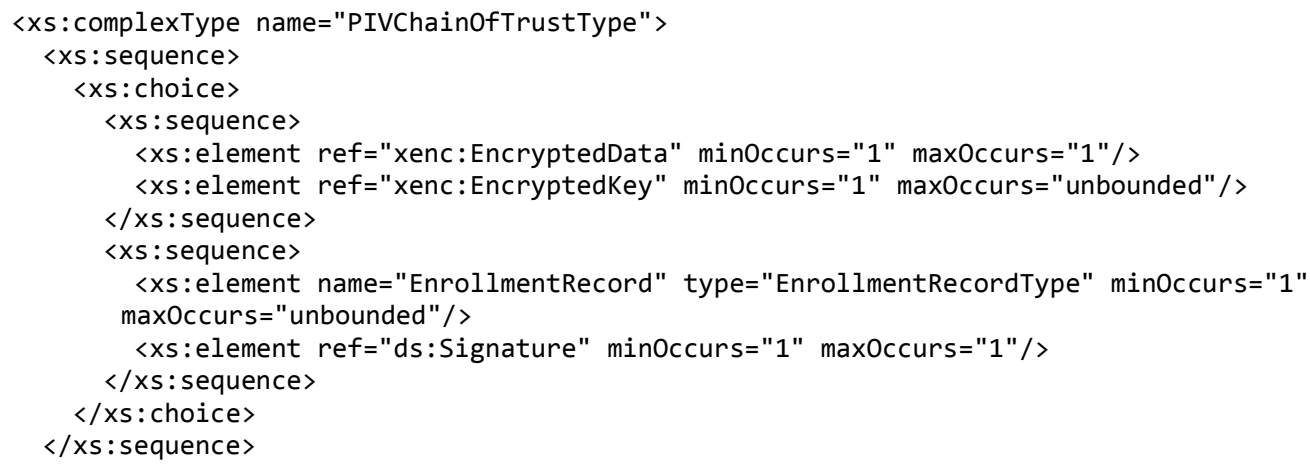


$</ x s$ : complexType $>$

The PIVChainOfTrust Type complex type offers a choice to either encrypt the PIV chain-of-trust records, or to leave the record unencrypted. PIV chain-of-trust records contain personally identifiable information (PII) of actual PIV cardholders and thus shall always be encrypted when in transit. The unencrypted sequence is intended to allow a recipient of the record to validate/test XML records as required. The recipient of the record should protect the records accordingly when not in use (i.e., data at rest protection).

Each instance of the <EnrollmentRecord> element contains the enrollment information captured by the PIV issuer for a single PIV cardholder. There is a minimum of one instance of this element, and there is no limit to the maximum number of $<$ EnrollmentRecord > elements that may be contained in the <PIVChainOfTrust $>$ element. This allows a single instance of the <PIVChainOfTrust $>$ element to contain several PIV cardholder records in an XML file.

Encrypted PIV chain-of-trust records are intended to include a confidentiality protection mechanism. The encrypted content and associated encryption details are defined by the XML Encryption Syntax and Processing specification [XMLEnc]. The <EncryptedData> Type attribute should be present and contain a value of http://www.w3.org/2001/04/xmlenc\#Element. The encryption method algorithm for the <EncryptedData> element should be set to: http://www.w3.org/2001/04/xmlenc\#aes256cbc.

The <xenc:EncryptedKey> element is defined in the [XMLEnc] standard and used to transport encrypted symmetric keys from the originator to a known recipient(s). The symmetric key value is always encrypted to the recipient(s). A public key from a PKI certificate should be used to encrypt the symmetric key. The sender should select an encryption certificate for the recipient issued under a policy that has been cross-certified with the Federal Bridge CA (FBCA) at the Medium Hardware or High Assurance Level. The sender should use http://www.w3.org/2001/04/xmlenc\#rsa-oaep-mgf1p as the key transport algorithm to encrypt the key. Each encrypted key should include a Recipient attribute that contains a hint as to which recipient the encrypted key value is intended for. An example value that could be used in the Recipient attribute is the distinguished name (DN) from the recipient's encryption certificate subject field.

A procedure for the exchange of the key encryption key is outside the scope of this document. Some example mechanisms for this exchange include the use of Secure/Multipurpose Internet Mail Extensions (S/MIME) email exchanges to share the encryption certificates, or a webpage that supports Hyper Text Transfer Protocol with Secure Sockets Layer (HTTPS) using an FPKI SSL certificate or an Extended Validation Secure Sockets Layer (EVSSL) certificate, where links to the encryption certificates for recipient federal agencies or service providers can be accessed and downloaded.

All PIV chain-of-trust records are signed using XML Signature. An XML Signature protects the integrity of the data and authenticates the issuer of the chain-of-trust. A valid 
PIV Card or a private key generated under the id-fpki-common-piv-contentSigning certificate policy should be used to sign the XML record.

\subsection{Complex Type EnrollmentRecordType}

The following schema fragment defines the EnrollmentRecordType complex type:

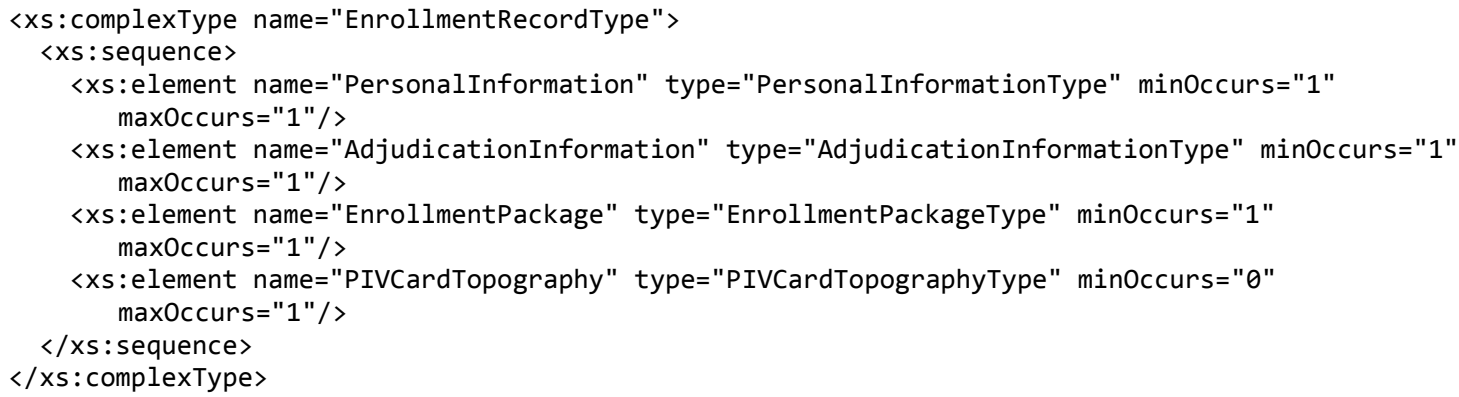

The EnrollmentRecordType complex type contains four elements carrying data required for the issuance of a PIV Card to the cardholder. The elements contained in the EnrollmentRecordType complex type are:

$<$ PersonalInformation>:

This element is an instance of the PersonalInformationType complex type and contains personally identifying information on the subject of the chain-oftrust enrollment record. The <PersonalInformation> element is a required element that appears once in the EnrollmentRecordType complex type.

$<$ AdjudicationInformation>:

This element is an instance of the AdjudicationInformation Type complex type and contains information on the status of the background investigation into the subject of the chain-of-trust enrollment record. The

$<$ AdjudicationInformation> element is a required element that appears once in the EnrollmentRecordType complex type.

$<$ EnrollmentPackage>:

This element is an instance of the EnrollmentPackageType complex type and contains biographic and biometric information on the subject of the chainof-trust enrollment record. The <EnrollmentPackage> element is a required element that appears once in the EnrollmentPackageType complex type.

$<$ PIVCardTopography $>$ :

This element is an instance of the PIVCardTopographyType complex type and contains information used for the physical printing of PIV Cards. The $<$ PIVCardTopography> element is an optional element. If used, it would appear once in the EnrollmentRecordType complex type.

The EnrollmentRecordType for an agency to agency record exchange use case would typically include the following types: 
- PersonalInformationType

- AdjudicationInformationType

- EnrollmentPackageType

The EnrollmentRecordType for an agency to service provider record exchange use case would typically include the following types:

- PersonalInformationType

- AdjudicationInformationType

- EnrollmentPackageType

- PIVCardTopographyType

As indicated above, the PIVCardTopographyType data element is more likely to be included in the service provider record exchange use case rather than the agency to agency record exchange use case. This is because a client agency needs to provide information about its agency and its new employees/contractors to be printed on the surface of each card. For the agency to agency record exchange, topographic information may not be needed as the receiving agency will use its own topographic elements when printing on the PIV Card.

\subsection{Complex Type PersonallnformationType}

The following schema fragment defines the PersonalInformationType complex type:

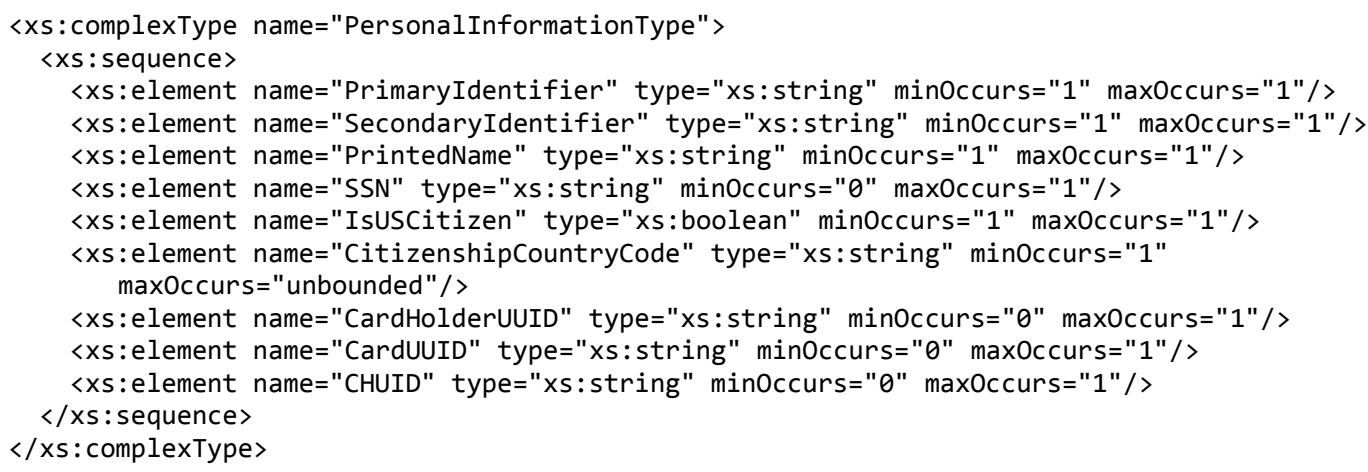

The CardUUID and CHUID type data element generally is not included in the agency to agency record exchange use case as the new agency will generate these values. For the agency to servicer provider record exchange, however, the client agency may wish to specify the CardUUID and CHUID for each PIV cardholder.

The PersonalInformationType complex type contains eight elements that describe the subject of the chain-of-trust enrollment record. Those elements are:

$<$ PrimaryIdentifier $>$ :

This element contains the Primary Identifier of the subject of the chain-oftrust enrollment record. The Primary Identifier is defined in [FIPS201] as the surnames or family names of the PIV cardholder. The <PrimaryIdentifier> element is always included in the PersonalInformationType complex type 
and appears only once. The data is provided in an xs:string format.

$<$ SecondaryIdentifier $>$ :

This element contains the Secondary Identifier of the subject of the chain-oftrust enrollment record. The Secondary Identifier is defined in [FIPS201] as pre-names or given names of the PIV Cardholder. The <SecondaryIdentifier > element is always included in the PersonalInformationType complex type and occurs only once. The data is provided in an xs:string format.

$<$ PrintedName $>$ :

This element contains the Printed Name of the subject of the chain-of-trust enrollment record. The Printed Name is a combination of the card holder's primary and secondary identifiers, as it is to be printed on the PIV Card according to [FIPS201]. The <PrintedName> element is always included in the PersonalInformationType complex type and only occurs once. The data is provided in an xs:string format.

$<\mathrm{SSN}>$ :

This element may contain the Social Security Number of the subject of the chain-of-trust enrollment record. ${ }^{1}$ The $\langle\mathrm{SSN}\rangle$ element is optional, and may occur only once if included in the PersonalInformationType complex type. The data is provided in an xs:string format.

$<$ IsUSCitizen>:

This element is required, occurs once in the PersonalInformationType complex type and contains a boolean value. If the subject of the chain-of-trust enrollment record is a U.S. Citizen, then the value is set to true. If the subject of the chain-of-trust enrollment record is not a U.S. Citizen, then the value is set to false. The data is provided in an xs:boolean format. If the $<$ IsUSCitizen> element is false, then at least one country is specified in $<$ CitizenshipCountryCode $>$.

$<$ CitizenshipCountryCode>:

This element is required if <IsUSCitizen> is set to false and optional if $<$ IsUSCitizen> is set to true. The element contains the countries of citizenship of the subject of the chain-of-trust enrollment record. The country code is formatted in an abbreviation (alpha-3 format), as documented in [ISO3166]. The <CitizenshipCountryCode> element may occur once for each citizenship the applicant possesses. The data is provided in an xs:string format.

${ }^{1}$ To eliminate unnecessary use of the SSN, whenever possible, departments and agencies should avoid using the <SSN> element. See the attachment to OMB M-07-16 [M-07-16], Section 2: "Reduce the Use of Social Security Numbers." 
$<$ CardHolderUUID $>$ :

The <CardHolderUUID $>$ element is optional, and occurs once when included in the PersonalInformationType complex type. The Cardholder UUID is is a persistent identifier for the cardholder. The data is provided in an xs:string format. <CardHolderUUID> can be used by Agency B to request record from Agency A. The user then re-connects with the record via a biometric match.

$<$ CardUUID $>$ :

The $<$ CardUUID $>$ element is optional, and occurs once when included in the PersonalInformationType complex type. It is envisioned that the $<$ CardUUID> will be used only in the agency to service provider record exchange use case to transfer the Card UUID to be stored on the PIV Card. The data is provided in an xs:string format. <CardUUID> can be used by Agency B to request a record from Agency A. The user then re-connects with the record via a biometric match.

$<$ CHUID $>$ :

This element is optional and if used should occur once when included in the PersonalInformationType complex type. It is envisioned that the <CHUID> will be used only in the agency to service provider use case to transfer the CHUID-specific details to be stored on the PIV Card. The data is provided in an xs:string format. <CHUID>'s FASC-N can be used by Agency B to request a record from Agency A. The user then re-connects with the record via a biometric match.

\subsection{Complex Type AdjudicationInformationType}

The following schema fragment defines the AdjudicationInformationType complex type:

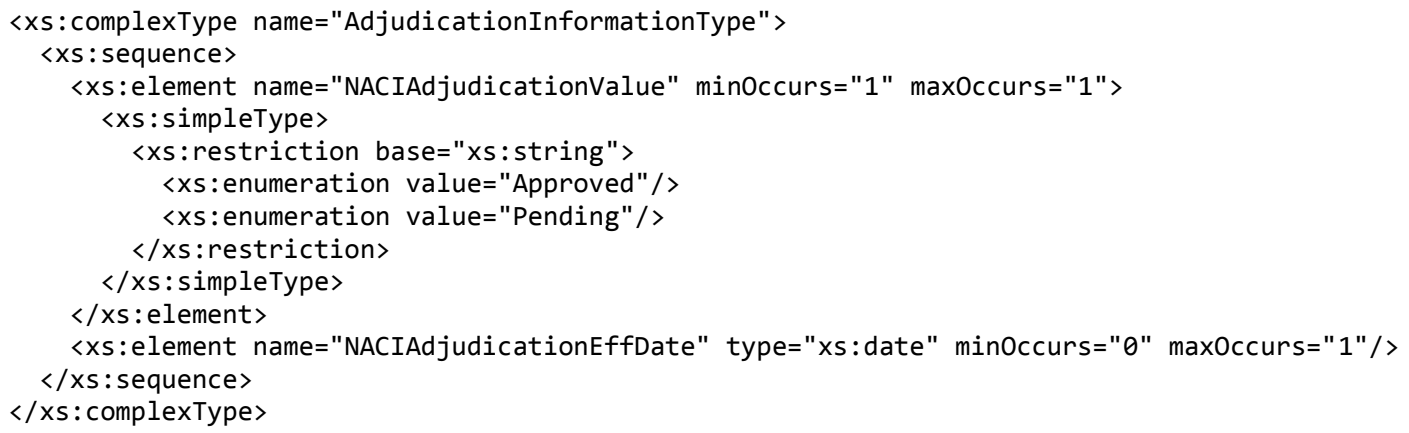

The AdjudicationInformationType complex type contains two elements that describe the status of the PIV background investigation of the subject of the chain-of-trust enrollment record. Those elements are:

$<$ NACIAdjudicationValue >:

This element is required, and occurs once in the

AdjudicationInformationType complex type. The element indicates the status of the National Agency Check with Inquiries (NACI) (or equivalent or 
higher) or Tier 1 or higher federal background investigation the PIV cardholder underwent. There are two permitted values based on the progress of the background investigation. The element is set to 'Approved' or 'Pending.' 'Approved' indicates a NACI (or equivalent or higher) or Tier 1 or higher federal background investigation has been completed. 'Pending' status indicates the Federal Bureau of Investigation (FBI) National Criminal History Check (NCHC) portion of the background investigation is completed and that NACI background check is in process, but has not yet received final adjudication. The data is provided in an xs:string format.

$<$ NACIAdjudicationEffDate>:

This element is optional, and occurs once when included in the AdjudicationInformation Type complex type. The element indicates the date the current status of the <NACIAdjudicationValue> element went into effect. The data is provided in an xs:date format.

\subsection{Complex Type EnrollmentPackageType}

The following schema fragment defines the EnrollmentPackageType complex type:

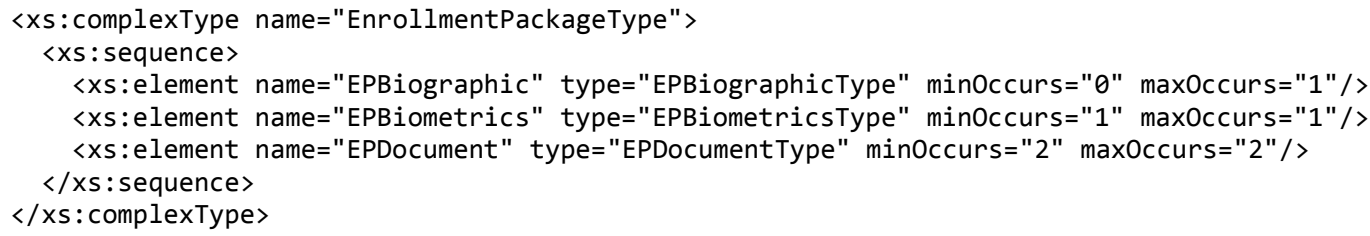

The EnrollmentPackage Type complex type contains three elements that identify the biographic and biometric properties of a PIV cardholder. The elements contained in the EnrollmentPackageType complex type are:

$<$ EPBiographic $>$ :

This element is an instance of the EPBiographicType complex type, containing biographic information on the subject of the chain-of-trust enrollment record as described in Section 3.8. The <EPBiographic $>$ element is an optional element and if present, appears once in the

EnrollmentPackageType complex type.

$<$ EPBiometrics $>$ :

This element is an instance of the EPBiometricsType complex type, containing biometric information on the subject of the chain-of-trust enrollment record as described in Section 3.9. The < EPBiometrics $>$ element is a required element that appears once in the EnrollmentPackageType complex type.

$<$ EPDocument $>$ :

This element is an instance of the EPDocumentType complex type as described in Section 3.10. The <EPDocument > element is a mandatory element and appears twice in the EnrollmentPackageType complex type. 
One instance of the <EPDocument $>$ element contains the primary identity source document as defined in [FIPS201]. The second instance of the $<$ EPDocument> element complex type contains the secondary identity source document as defined in [FIPS201].

\subsection{Complex Type EPBiographicType}

The following schema fragment defines the EPBiographicType complex type:

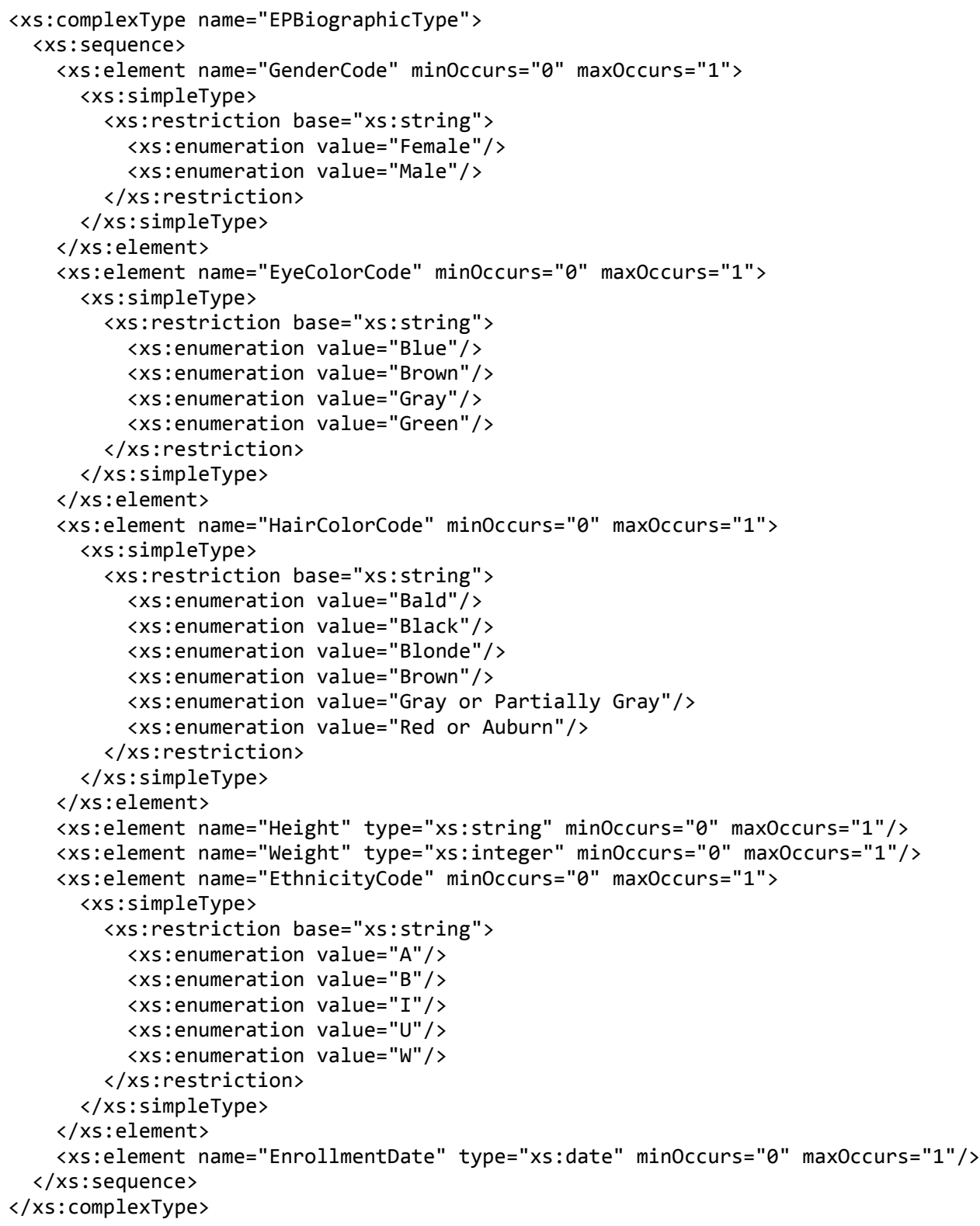

The EPBiographicType complex type contains six optional elements that describe the biographical data of the subject of the chain-of-trust enrollment record. Values for EPBiographicType complex type are based on the FBI's Electronic Biometric Transmission specification [EBTS]. Those elements are: 
$<$ GenderCode > :

This element contains the gender of the subject of the chain-of-trust enrollment record. The <GenderCode> element is optional and occurs once when included in the EPBioGraphicType complex type. The <GenderCode> element is set to Female or Male. The data is provided in an xs:string format.

$<$ EyeColorCode $>$ :

This element contains the eye color of the subject of the chain-of-trust enrollment record. The <EyeColorCode $>$ element is optional and occurs once when included in the EPBioGraphicType complex type. The $<$ EyeColorCode> element is selected from the following values: Blue, Brown, Gray or Green. The data is provided in an xs:string format.

\section{$<$ HairColorCode>:}

This element contains the hair color of the subject of the chain-of-trust enrollment record. The <HairColorCode> element is optional and occurs once when included in the EPBioGraphicType complex type. The $<$ HairColorCode> element is selected from the following values: Bald, Black, Blonde, Brown, Gray or Partially Gray, Red or Auburn. The data is provided in an xs:string format.

$<$ Height $>$ :

This element contains the physical height of the subject of the chain-of-trust enrollment record. If reported in feet and inches, the first (leftmost) digit is used to show feet while the two rightmost digits are used to show the inches between 00 and 11 . If reported in inches, then the leftmost character is ' $N$ ' followed by two digits. The <Height> element is optional and occurs once when included in the EPBioGraphicType complex type. The data is provided in an xs:string format.

<Weight:>:

This element contains the physical weight of the subject of the chain-of-trust enrollment record. The subject's weight in pounds is entered. All weights in excess of 499 pounds will be set to 499 . The <Weight> element is optional and occurs once when included in the EPBioGraphicType complex type. The data is provided in an xs:string format.

\section{$<$ EthnicityCode>:}

This element contains the ethnicity of the subject of the chain-of-trust enrollment record. The <EthnicityCode> element is optional and occurs once when included in the EPBioGraphicType complex type. The $<$ EthnicityCode> element is selected from the following values: A, B, I, U, or W. 
Table 2: Ethnicity Codes

\begin{tabular}{|l|c|}
\hline If Subject Is & Enter Code \\
\hline $\begin{array}{l}\text { Chinese, Japanese, Filipino, Korean, Polynesian, Indian, Indonesian, } \\
\text { Asian Indian, Samoan, or any other Pacific Islander }\end{array}$ & A \\
\hline A person having origins in any of the black racial groups of Africa & B \\
\hline $\begin{array}{l}\text { American Indian, Eskimo, or Alaskan native, or a person having } \\
\text { origins in any of the 48 contiguous states of the United States or } \\
\text { Alaska who maintains cultural identification through tribal affiliation } \\
\text { or community recognition }\end{array}$ & I \\
\hline $\begin{array}{l}\text { Of indeterminable race } \\
\text { Caucasian, Mexican, Puerto Rican, Cuban, Central or South } \\
\text { American, or other Spanish culture or origin, Regardless of race }\end{array}$ & U \\
\hline
\end{tabular}

$<$ EnrollmentDate $>$ :

This element contains the date the subject of the chain-of-trust enrollment record enrolled for a PIV Card. The <EnrollmentDate> element is optional and occurs once when included in the EPBioGraphicType complex type. The data is provided in an xs:date format.

\subsection{Complex Type EPBiometricsType}

The following schema fragment defines the EPBiometricsType complex type:

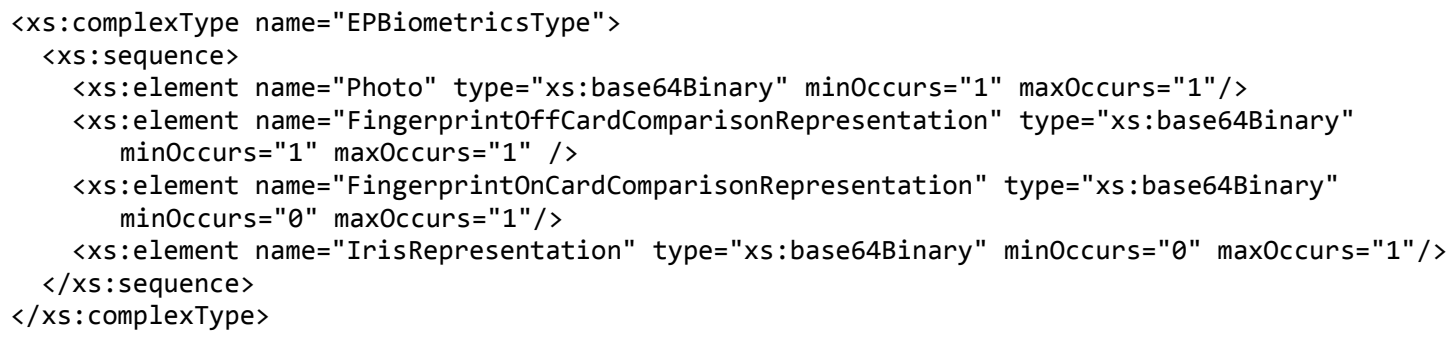

The EPBiometrics complex type contains information related to the biometric data of the subject of the chain-of-trust enrollment record, as captured during PIV enrollment including the primary and secondary fingerprint representation. The four elements supported in the EPBiometrics complex type are:

$<$ Photo $>$ :

This element is required. The <Photo> element contains the facial image collected during the enrollment process, as documented in [SP800-76] Biometric Specifications for Personal Identity Verification. The <Photo> element includes the CBEFF Header, the INCITS 385 profile for PIV facial images, and the CBEFF signature. The facial image is used to both print the image on the card and as the digital representation stored in the PIV Card. The data is provided in an xs:base64Binary format. 
<FingerprintsOffCardComparisonRepresentation>:

This element is mandatory and occurs only once. The

$<$ FingerprintsOffCardComparisonRepresentation> element contains the fingerprint data collected during the enrollment process and is used for offcard comparison, as documented in [SP800-76]. The

$<$ FingerprintsOffCardComparisonRepresentation> element includes the CBEFF Header, the INCITS 378 Minutiae, and the CBEFF signature. The data is provided in an xs:base64Binary format.

<FingerprintsOnCardComparisonRepresentation>:

This element is optional and if used occurs once.

$<$ FingerprintsOnCardComparisonRepresentation> element is only used if the $<$ FingerprintsOnCardComparisonRepresentation> element uses different fingers representation than in

$<$ FingerprintsOffCardComparisonRepresentation> element. If the fingers for on-card and off-card comparison representation are identical, on-card comparison minutia templates representation can be created using the data stored in the <FingerprintsOffCardComparsionRepresentation> element. If used, the <FingerprintsOnCardComparisonRepresentation> includes the CBEFF Header, the ISO/IEC 19794-2:2011 minutiae, and the CBEFF signature, as documented in [SP800-76]. The data is provided in an xs:base64Binary format.

$<$ IrisRepresentation>:

This element is optional and if used occurs once. The <IrisRepresentation> element contains the iris image representation collected during the enrollment process, as documented in [SP800-76]. The <IrisRepresentation> element includes the CBEFF Header, ISO 19794-6 iris, and the CBEFF signature. The data is provided in an xs:base64Binary format.

\subsection{Complex Type EPDocumentType}

The following schema fragment defines the EPDocumentType complex type:

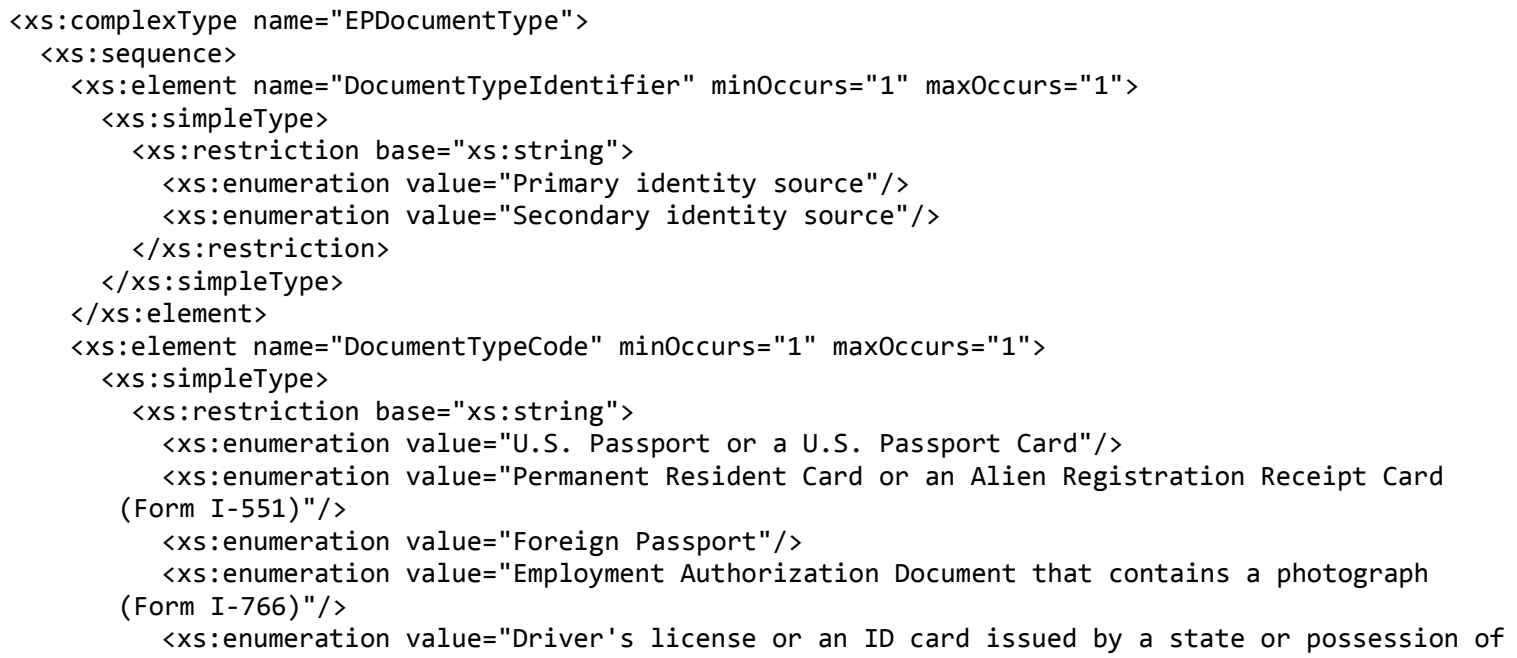




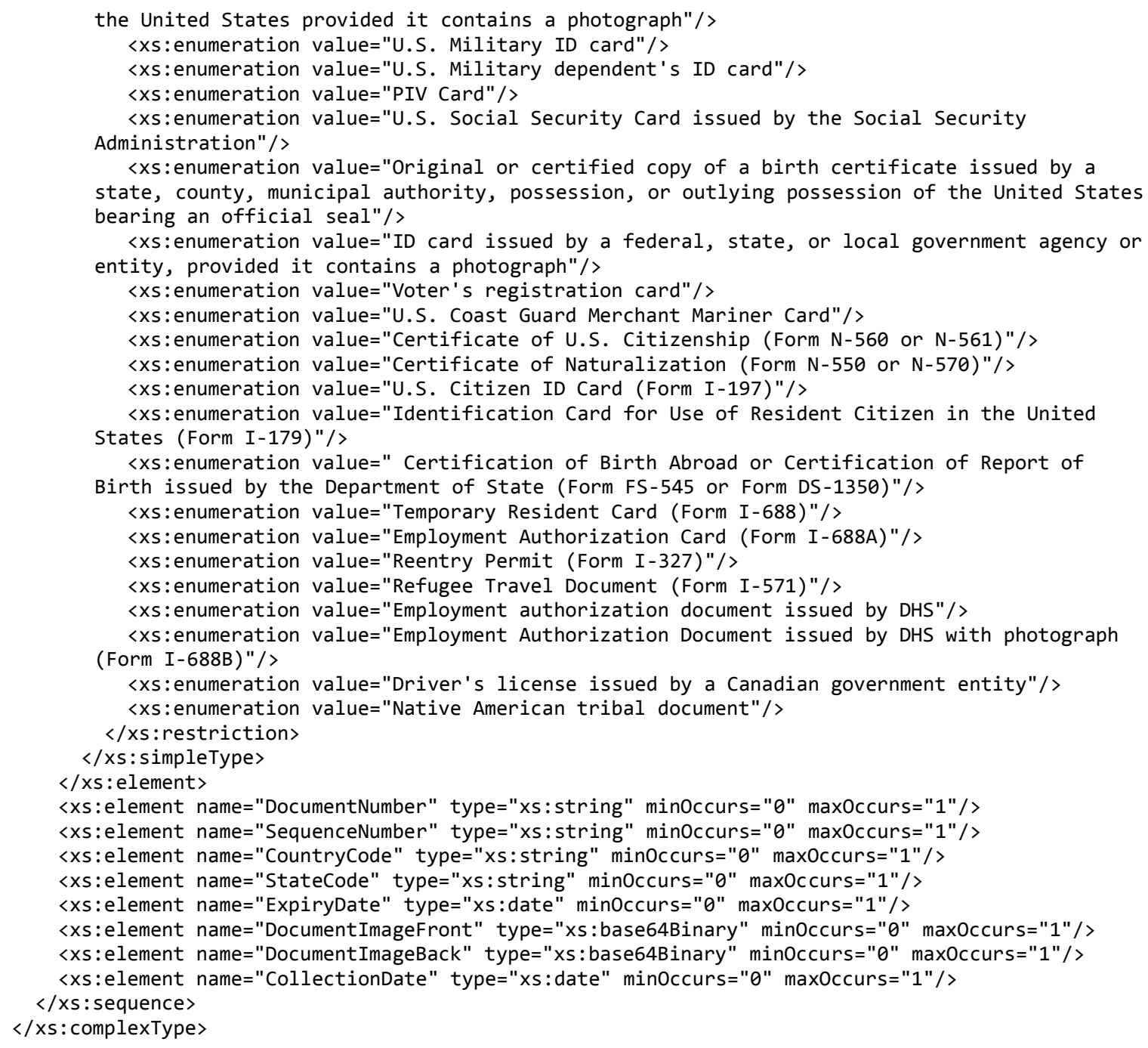

The EPDocumentType complex type contains information related to the identity source document presented by the subject of the PIV chain-of-trust enrollment record during the enrollment process. There are two instances of the type to specify primary and secondary identity source documents. The ten elements supported in each EPDocument Type complex type are:

$<$ DocumentTypeIdentifier>:

This element is required and occurs once. The element represents the identity source document presented during the [FIPS201] identity proofing process.

The <DocumentTypeIdentifier> element indicates either: the Primary identity source document or the Secondary identity source document as the value. The data is provided in an xs:string format.

$<$ DocumentTypeCode>:

This element is required and occurs once. The <DocumentTypeCode> element indicates the type of document verified during the enrollment of the PIV cardholder. The data is provided in an xs:string format. The 
<DocumentTypeCode> element is selected from the following two bulleted lists of permitted values, as documented in [FIPS201]:

For one of the EPDocumentType instances, the identity source document is a primary identity source document and one of the following values should be used:

- U.S. Passport or a U.S. Passport Card;

- Driver's license or an ID card issued by a state or possession of the United States provided it contains a photograph;

- Permanent Resident Card or an Alien Registration Receipt Card (Form I-551);

- Foreign passport;

- Employment Authorization Document that contains a photograph (Form I-766);

- U.S. Military ID card;

- U.S. Military dependent's ID card; or

- PIV Card.

For the other EPDocumentType instance, the secondary identity source document is indicated and the following values should be used:

- U.S. Social Security Card issued by the Social Security Administration;

- Original or certified copy of a birth certificate issued by a state, county, municipal authority, possession, or outlying possession of the United States bearing an official seal;

- ID card issued by a federal, state, or local government agency or entity, provided it contains a photograph;

- Voter's registration card;

- U.S. Coast Guard Merchant Mariner Card;

- Certificate of U.S. Citizenship (Form N-560 or N-561);

- Certificate of Naturalization (Form N-550 or N-570);

- U.S. Citizen ID Card (Form I-197);

- Identification Card for Use of Resident Citizen in the United States (Form I-179);

- Certification of Birth Abroad or Certification of Report of Birth issued by the Department of State (Form FS-545 or Form DS-1350);

- Temporary Resident Card (Form I-688);

- Employment Authorization Card (Form I-688A);

- Reentry Permit (Form I-327);

- Refugee Travel Document (Form I-571);

- Employment authorization document issued by DHS;

- Employment Authorization Document issued by DHS with photograph (Form I-688B);

- Driver's license issued by a Canadian government entity; or 
- Native American tribal document.

<DocumentNumber>:

This element is optional, and if used occurs once. The element indicates the document number from the identity source document verified during enrollment. The data is provided in an xs:string format.

$<$ SequenceNumber $>$ :

This element is optional, and if used occurs once. The element indicates the sequence number from the identity source document verified during enrollment. The data is provided in an xs:string format.

$<$ CountryCode $>$ :

This element is optional and if used occurs once. The element indicates the country that issued the identity source document verified during the PIV enrollment process. The country code is formatted in an abbreviation (alpha-3 format), as documented in [ISO3166]. The data is provided in an xs:string format.

$<$ StateCode $>$ :

This element is optional and if used occurs once. The element indicates the US state that provided the identity source document verified during enrollment. The state name is formatted as a two letter abbreviation, as documented by the United States Postal Service [USPS-Appendix B]. The data is provided in an xs:string format.

$<$ ExpiryDate $>$ :

This element is optional and if used occurs only once. The element indicates the date the identity source document verified during enrollment is set to expire. The data is provided in an xs:date format.

$<$ DocumentImageFront $>$ :

This element is optional and if used occurs only once. The <DocumentImageFront> element contains a scanned JPEG or PDF image of the front of the identity source document verified during enrollment. The data is provided in an xs:base64Binary format.

$<$ DocumentImageBack $>$ :

This element is optional and if used occurs only once. The $<$ DocumentImageBack> element contains a scanned JPEG or PDF image of the back of the identity source document verified during enrollment. The data is provided in an xs:base64Binary format.

$<$ CollectionDate>:

This element is optional and if used occurs only once. The element indicates the date the identity source document was collected and verified by an enrollment official. The data is provided in an xs:date format. 


\subsection{Complex Type PIVCardTopographyType}

\section{The following schema fragment defines the PIVCardTopographyType complex type:}

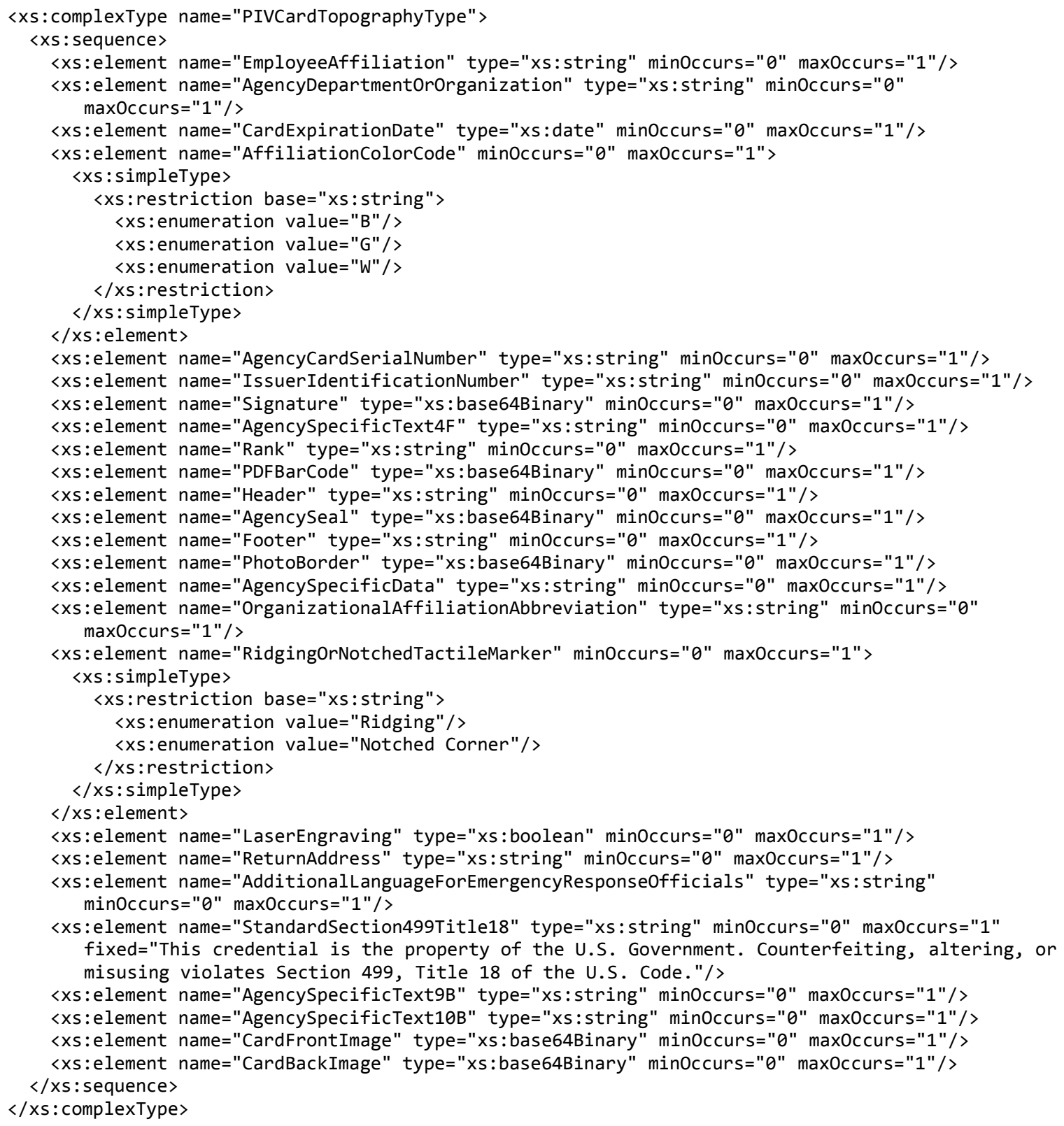

The PIVCardTopography complex type contains information used for printing the front and back of the PIV Card as described in [FIPS201] $]^{2}$. The twenty-three elements supported in the PIVCardTopography complex type are:

\footnotetext{
${ }^{2}<$ PrintedName $>$ in the PersonalInformationType can be used to print the name of the subject on the front of the card.
} 
$<$ EmployeeAffiliation>:

This element is optional and if used occurs only once. The element contains the employee affiliation to be printed on the card. Some examples of employee affiliation are "Employee," "Contractor," "Active Duty," and "Civilian." The <EmployeeAffiliation> element is the value for Zone 8F on the front of the PIV Card. The data is provided in an xs:string format.

<AgencyDepartmentOrOrganization>:

This element is optional and if used occurs only once. The element contains the organizational affiliation of the cardholder. The $<$ AgencyDepartmentOrOrganization> element is the value for Zone $10 \mathrm{~F}$ on the front of the PIV Card. The data is provided in an xs:string format.

$<$ CardExpirationDate $>$ :

This element optional and if used occurs only once. The element contains the date the PIV Card is set to expire. The <CardExpirationDate> element is the value for Zone 14F on the front of the PIV Card. The data is provided in an xs:date format.

$<$ AffiliationColorCode >:

This element is optional and if used occurs only once. The

<AffiliationColorCode> element is the value for Zone $18 \mathrm{~F}$ on the front of the PIV Card. This value also indicates the color that is to be used in Zone 15F and as a background color for Zone $2 \mathrm{~F}$ (name). The <AffiliationColorCode> element is selected from the following values: B, G, or W. B indicates Blue for Foreign National, $\mathrm{G}$ indicates Green for Contractors, and $\mathrm{W}$ indicates White for Government Employee. The data is provided in an xs:string format.

<AgencyCardSerialNumber>:

This element is optional and if used occurs only once. The element contains the unique serial number from the issuing department or agency. The format shall be at the discretion of the issuing department or agency. The $<$ AgencyCardSerialNumber> element is the value for Zone 1B on the back of the PIV Card. The data is provided in an xs:string format.

<IssuerIdentificationNumber>:

This element is optional and if used occurs only once. The element consist of six characters for the department code, four characters for the agency code, and a five-digit number that uniquely identifies the issuing facility within the department or agency. The <IssuerIdentificationNumber> element is the value for Zone 2B on the back of the PIV Card. The data is provided in an xs:string format.

$<$ Signature $>$ :

This element is optional and if used occurs only once. The element contains a JPEG image of the cardholder's signature. The <Signature > element is the 
value for Zone 3F on the front of the PIV Card. The data is provided in an xs:base64Binary format.

$<$ AgencySpecificText4F>:

This element is optional and if used occurs only once. The element contains text for printing agency specific requirements on the PIV Card, such as employee status. The <AgencySpecificText4F> element is the value for Zone $4 \mathrm{~F}$ on the front of the PIV Card. The data is provided in an xs:string format.

$<$ Rank $>$ :

This element is optional and if used occurs only once. The element contains text for the cardholder's rank. The format of the text is at the department or agency's discretion. The <Rank> element is the value for Zone 5F on the front of the PIV Card. The data is provided in an xs:string format.

\section{$<$ PDFBarCode $>$ :}

This element is optional and if used occurs only once. The element contains a Portable Data File (PDF) Two-Dimensional Bar Code JPEG image. The $<$ PDFBarCode $>$ element is the value for Zone $6 \mathrm{~F}$ on the front of the PIV Card. The data is provided in an xs:base64Binary format.

$<$ Header $>$ :

This element is optional and if used occurs only once. If used the element should contain either the text "United States Government", or departments and agencies may also choose to use this text for other department or agencyspecific information, such as identifying a federal emergency responder role. The <Header $>$ element is the value for Zone 9F on the front of the PIV Card. The data is provided in an xs:string format.

$<$ AgencySeal >

This element is optional and if used occurs only once. The element contains a JPEG image of the seal selected by the issuing department, agency, or organization for printing on the PIV Card. The <AgencySeal> element is the value for Zone $11 \mathrm{~F}$ on the front of the PIV Card. The data is provided in an xs:base64Binary format.

\section{$<$ Footer $>$ :}

This element is optional and if used occurs only once. If used the element should contain the location label for the Federal Emergency Response Official (FERO) identification. A department or agency may print "Federal Emergency Response Official", preferably in white lettering on a red background on the PIV Card. Departments and agencies may also use the $<$ Footer> element to further identify the federal emergency respondent's official role. Some examples of official roles are "Law Enforcement," "Fire Fighter," and "Emergency Response Team (ERT)." When Zone 15F indicates foreign national affiliation and the department or agency does not need to 
highlight emergency response official status, the <Footer > element may be used to denote the country or countries of citizenship. If so used, the department or agency shall print the country name or the three-letter country abbreviation (alpha-3 format) in accordance with ISO 3166-1, Country Codes [ISO3166]. The <Footer> element is the value for Zone 12F on the front of the PIV Card. The data is provided in an xs:string format.

$<$ PhotoBorderPattern $>$ :

This element is optional and if used occurs only once. The element contains a JPEG image of the photo border. A border may be used with the photo to further identify employee affiliation. This border may be used in conjunction with Zone $15 \mathrm{~F}$ to enable departments and agencies to develop various employee categories. The photo border shall not obscure the photo. The border may be a solid or patterned line. For solid and patterned lines, red shall be reserved for emergency response officials, blue for foreign nationals, and green for contractors. All other colors may be used at the department or agency's discretion. The <PhotoBorderPattern> element is the value for Zone $16 \mathrm{~F}$ on the front of the PIV Card. The data is provided in an xs:base64Binary format.

$<$ AgencySpecificData>:

This element is optional and if used occurs only once. In cases in which other defined optional elements are not used, the <AgencySpecificData> element may be used for other department or agency-specific information and is set to the value of Zone 17F on the front of the PIV Card. The data is provided in an $\mathrm{xs}$ :string format.

$<$ OrganizationalAffiliationAbbreviation>:

This element is optional and if used occurs only once. The element contains the organizational affiliation abbreviation for printing on the PIV Card. The $<$ OrganizationalAffiliationAbbreviation> element is the value for Zone 20F on the front of the PIV Card. The data is provided in an xs:string format.

$<$ RidgingOrNotchedTactileMarker >:

This element is optional and if used occurs only once. The element indicates if the PIV Card should incorporate edge ridging or a notched corner to indicate card orientation. The <RidgingOrNotchedTactileMarker $>$ element is selected from the following values: "Ridging" or Notched Corner". The $<$ RidgingOrNotchedTactileMarker $>$ element is the value for Zone $21 \mathrm{~F}$ on the front of the PIV Card. The data is provided in an xs:string format.

$<$ LaserEngraving >

This element is optional and if used occurs only once. The element indicates if the PIV Card should include tactilely discernible marks created using laser engraving to indicate card orientation. The <LaserEngraving> element is set to true if laser engraving should be applied to the PIV Card and set to false if 
laser engraving should not be applied to the PIV Card. The <LaserEngraving> element is the value for Zone $22 \mathrm{~F}$ on the front of the PIV Card. The data is provided in an xs:boolean format.

$<$ ReturnAddress $>$ :

This element is optional and if used occurs only once. The element contains the "return if lost" language placed on the back of the PIV Card. The $<$ ReturnAddress > element is the value for Zone 4B on the back of the PIV Card. The data is provided in an xs:string format.

<AdditionalLanguageForEmergencyResponseOfficials>:

This element is optional and if used occurs only once. The element contains text if the departments and agencies choose to provide additional information to identify emergency response officials or to better identify the cardholder's authorized access. The <AdditionalLanguageForEmergencyResponseOfficials> element is the value for Zone 6B on the back of the PIV Card. The data is provided in an xs:string format.

$<$ StandardSection499Title18>:

This element is optional and if used occurs only once. The element contains the fixed text for the standard Section 499, Title 18, language warning against counterfeiting, altering, or misusing the PIV Card. The $<$ StandardSection499Title18> element is the value for Zone 7B on the back of the PIV Card. The data is provided in an xs:string format.

\section{$<$ AgencySpecificText9B>:}

This element is optional and if used occurs only once. The element contains text for printing agency specific requirements on the PIV Card. Departments and agencies are encouraged to use this area prudently and minimize printed text to that which is absolutely necessary. The <AgencySpecificText9B > element is the value for Zone 9B on the back of the PIV Card. The data is provided in an xs:string format.

\section{$<$ AgencySpecificText10B>:}

This element is optional and if used occurs only once. The element contains text for printing agency specific requirements on the PIV Card. Departments and agencies are encouraged to use this area prudently and minimize printed text to that which is absolutely necessary. The <AgencySpecificText10B> element is the value for Zone 10B on the back of the PIV Card. The data is provided in an xs:string format.

\section{$<$ CardFrontImage $>$ :}

This element is optional and if used occurs only once. The element contains a JPEG image of how the front of the PIV Card should appears when printed. The data is provided in an xs:base64Binary format. 
$<$ CardBackImage $>$ :

This element is optional and if used occurs only once. The element contains a JPEG image of how the back of the PIV Card should appears when printed. The data is provided in an xs:base64Binary format. 


\section{Chain-of-trust Record Encryption in Transit}

The confidentiality of the chain-of-trust record is to be protected at all times by both the producing and consuming organizations. In addition to encrypting the chain-of-trust record in accordance with XML encryption, as documented in Section 3.3 for PIVChainOfTrustType, the chain-of-trust records should be encrypted in transit between the chain-of-trust producer and the chain-of-trust consumer. Some example mechanisms for chain-of-trust transmission include but are not limited to: FTPS, HTTPS secured web services, and out of band mechanisms such as S/MIME secure email. The encryption used shall be compliant with the [FIPS140] standard. 


\section{$5 \quad$ Log and Historical Data}

Logs and historical data are maintained by the original issuer and are not transferred when exchanging PIV Card enrollment records. These log and historical data were created by the original issuer and are part of the chain-of-trust record. Log and historical data contain information that correlates the subject of a chain-of-trust record to logs of identity proofing, registration (enrollment) and maintenance activities of a cardholder. This includes information about the officer who took the action, what action was taken, and when and where the action occurred. Some examples of log activities maintained in the chain-of-trust record log are: initial PIV Cardholder registration, enrollment, and issuance, change of name and subsequent re-issuance, loss of card and subsequent reissuance. 


\section{Appendix A-XML Schema}

This appendix contains the chain of trust XML schema, which can also be download at http://csrc.nist.gov/schema/piv/chain-of-trust/pivchain-of-trust-1.0.xsd.

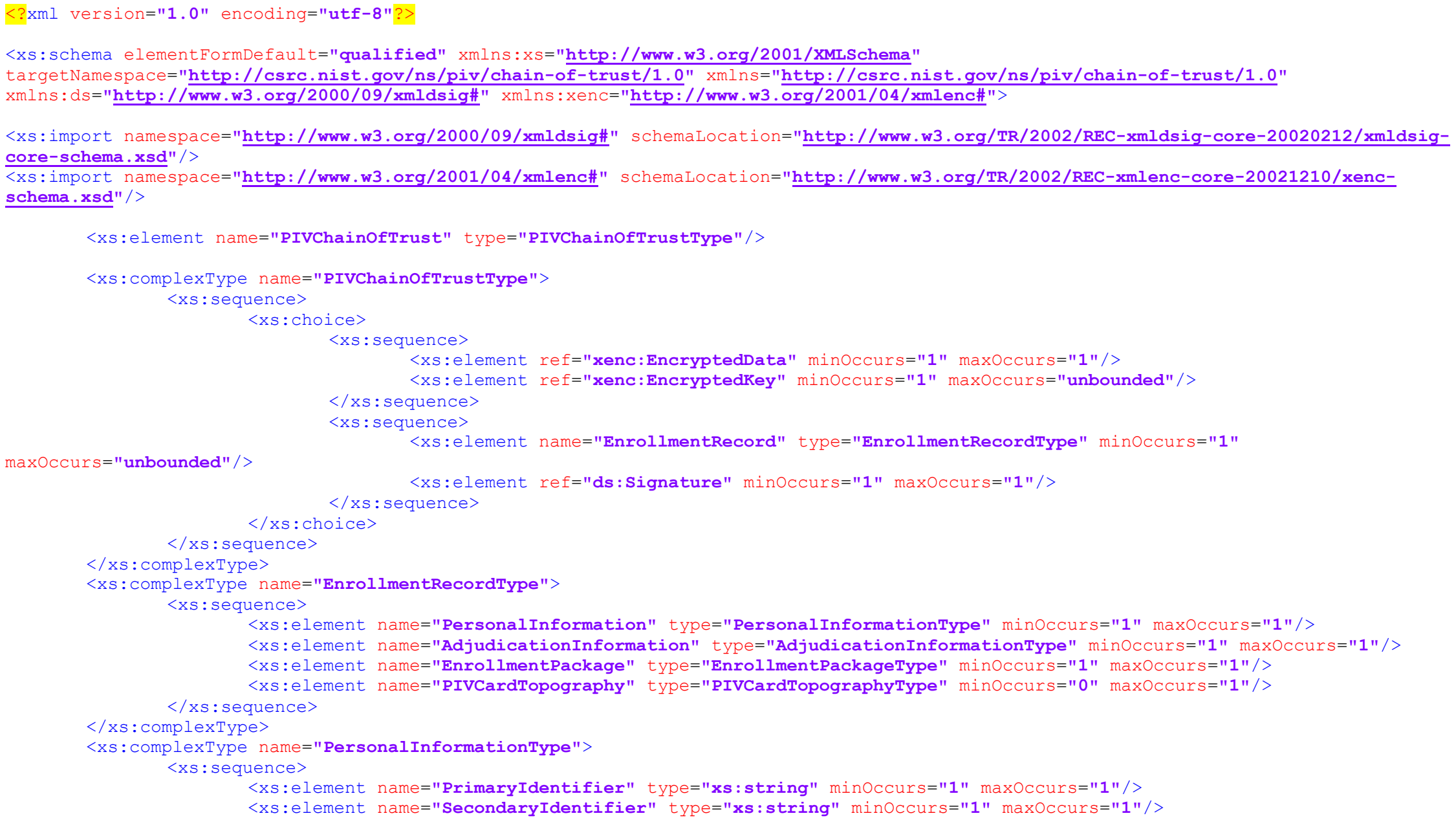


<xs:element name="PrintedName" type="xs:string" minoccurs="1" maxOccurs="1"/>

$<x s$ :element name="SSN" type="xs:string" minOccurs="0" maxOccurs="1"/>

$<x s$ :element name="Isuscitizen" type="xs:boolean" minoccurs="1" maxOccurs="1"/>

<xs:element name="CitizenshipCountryCode" type="xs:string" minOccurs="1" maxOccurs="unbounded"/>

$<x s: e l e m e n t$ name="CardHolderUUID" type="xs:string" minOccurs="0" maxOccurs="1"/>

<xs:element name="CardUUID" type="xs:string" minOccurs="0" maxOccurs="1"/>

$</ x s$ : sequence

$<x \mathrm{~s}:$ lement name="CHUID" type="xs:string" minOccurs="0" maxOccurs="1"/>

$</ x s$ : complexType

$<x s$ : complexType name="AdjudicationInformationType">

$<x s$ : sequence>

$<x s:$ element name="NACIAdjudicationValue" minoccurs="1" maxOccurs="1"> $<x$ : simpleType>

$<x s$ :restriction base="xs:string">

$<x$ : enumeration value="Approved" />

$</ x$ : simpleType

$</ x s:$ element $>$

<xs:element name="NACIAdjudicationEffDate" type="xs:date" minOccurs="0" maxOccurs="1"/>

$</ x s$ : sequence $>$

$</ x$ : complexType $>$

$<x s$ : complexType name="EnrollmentPackageType" >

$<x$ : sequence>

<XS:element name="EPBiographic" type="EPBiographicType" minOccurs="0" maxOccurs="1"/>

$<x s: e l e m e n t$ name="EPBiometrics" type="EPBiometricsType" minOccurs="1" maxOccurs="1"/>

$<x s: e l e m e n t$ name="EPDocument" type="EPDocumentType" minoccurs="2" maxOccurs="2"/> $\langle/ x s$ : sequence $>$

$</ x$ : complextype $>$

$<x s:$ complexType name="EPBiographicType">

$\langle x s$ : sequence $>$

<xs:element name="GenderCode" minoccurs="0" maxoccurs="1">

$<x s:$ impleType>

<xs:restriction base="xs:string">

$<x s$ :enumeration value="Female"/>

$<x$ : enumeration value="Male"/>

$</ x s$ :restriction $>$

$</ x s:$ element $>$

/xs: simpleType

$<x s:$ element name="EyeColorCode" minOccurs="0" maxOccurs="1">

<xs:simpleType>

<xs:restriction base="xs:string">

$<x s$ : enumeration value="Blue" />

$<x$ : enumeration value="Brown"/>

$<x$ : enumeration value="Gray" $/>$ 


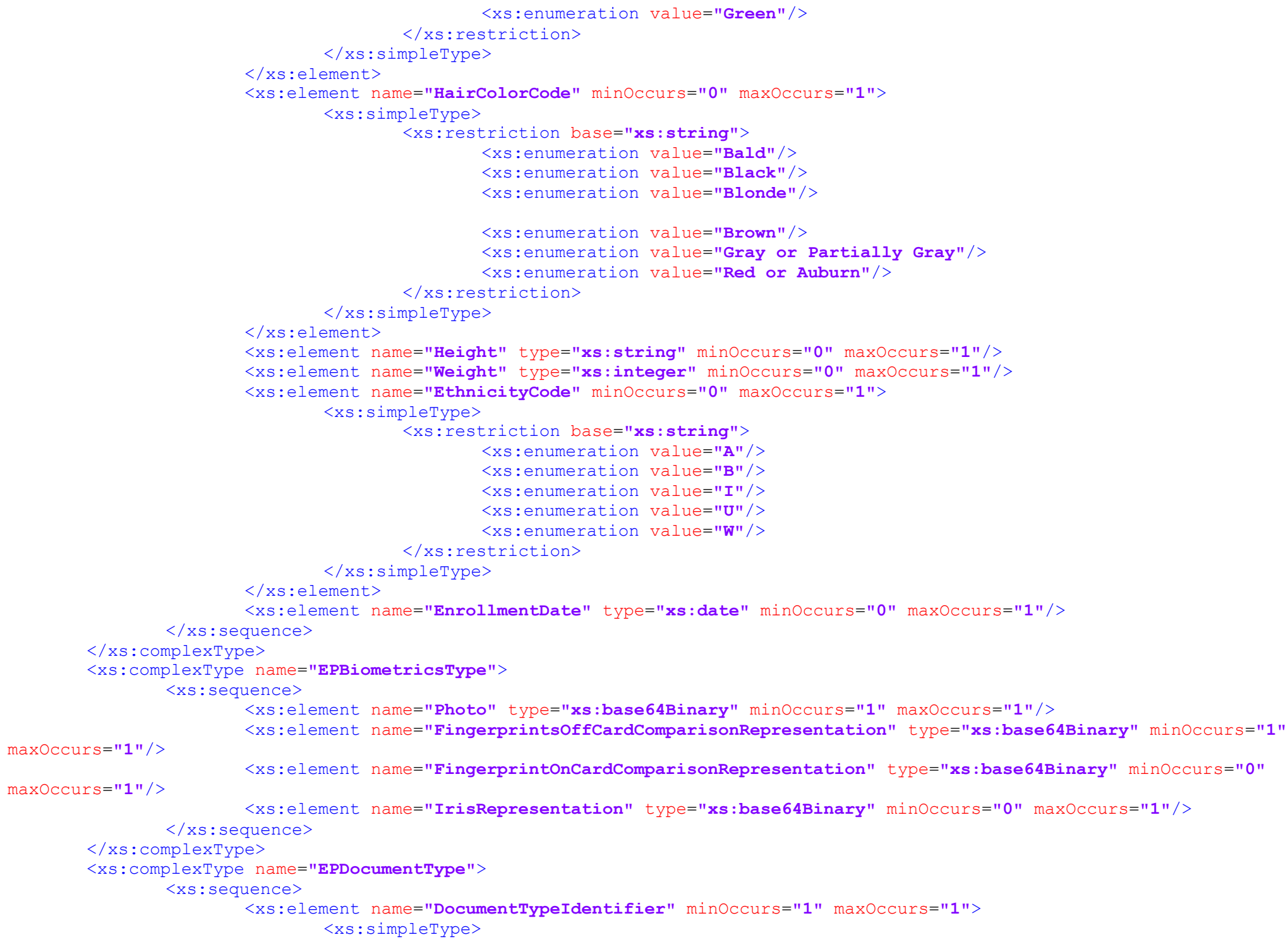




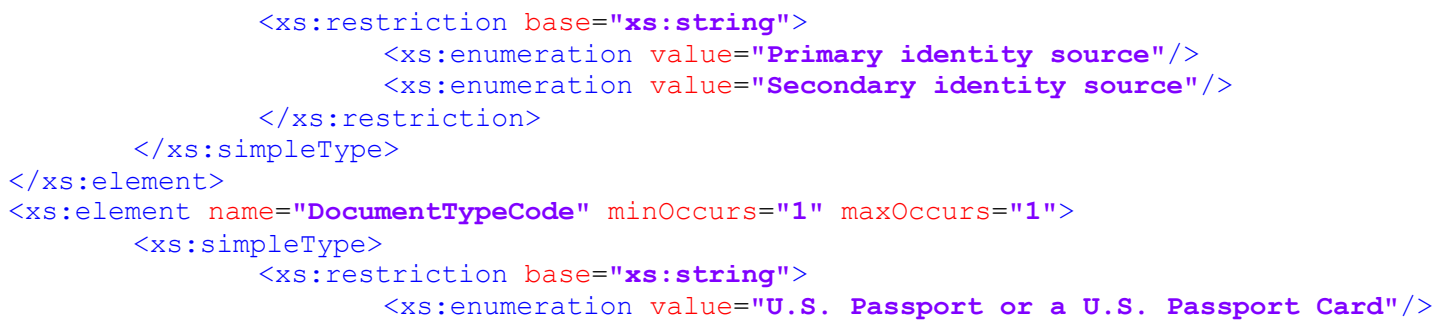

(Form I-551) "/>

$<x s$ : enumeration value="Permanent Resident Card or an Alien Registration Receipt Card

I-766) $" />$

$<x s$ :enumeration value="Foreign Passport" / >

<xs:enumeration value="Employment Authorization Document that contains a photograph (Form

the United States provided it contains a photograph"/>

$<$ Xs:enumeration value="U.S. Military ID card"/>

$<x$ : enumeration value="U.S. Military dependent's ID card"/>

$<x$ : enumeration value="PIV Card"/>

Administration" />

state, county, municipal authority, possession, or outlon value="Original or certified copy of a birth certificate iss

<xs:enumeration value="ID card issued by a federal, state, or local government agency or entity, provided it contains a photograph"/>

$<x$ : enumeration value="Voter's registration card"/>

$<$ xs:enumeration value="U.S. Coast Guard Merchant Mariner Card"/>

<xs:enumeration value="Certificate of U.S. Citizenship (Form N-560 or N-561)" / >

<xs:enumeration value="Certificate of Naturalization (Form N-550 or N-570)"/>

$<x$ : enumeration value="U.S. Citizen ID Card (Form I-197)"/>

<xs:enumeration value="Identification Card for Use of Resident Citizen in the United

States (Form I-179)"/>

<xs:enumeration value=" Certification of Birth Abroad or Certification of Report of Birth issued by the Department of State (Form FS-545 or Form DS-1350) "/>

<Xs: enumeration value="Temporary Resident Card (Form I-688)"/>

$<\mathrm{xs}$ : enumeration value="Employment Authorization Card (Form I-688A)"/>

$<x$ : enumeration value="Reentry Permit (Form I-327)" />

$<x$ : enumeration value="Refugee Travel Document (Form I-571)"/>

<xs:enumeration value="Employment authorization document issued by DHS"/>

(Form I-688B) " /

<xs:enumeration value="Employment Authorization Document issued by DHS with photograph

<xs:enumeration value="Driver's license issued by a Canadian government entity"/> $</ x$ : restriction>

$</ x s:$ impletype>

$</ x s$ :element $>$ 
$<x S: e l e m e n t$ name="DocumentNumber" type="xs:string" minOccurs="1" maxOccurs="1"/> $<x s: e l e m e n t$ name="SequenceNumber" type="xs:string" minoccurs="0" maxOccurs="1"/>

$<x s: e l e m e n t$ name="CountryCode" type="xs:string" minoccurs="0" maxOccurs="1"/>

$<x s: e l e m e n t$ name="StateCode" type="xs:string" minoccurs="0" maxOccurs="1"/>

$<x s: e l e m e n t$ name="ExpiryDate" type="xs:date" minoccurs="0" maxoccurs="1"/>

$<x s: e l e m e n t$ name="DocumentImageFront" type="xs:base64Binary" minOccurs="0" maxOccurs="1"/>

$<x s:$ element name="DocumentImageBack" type="xs:base64Binary" minOccurs="0" maxOccurs="1"/>

$</ x s$ : $<$ xs:el

$</ x s$ : complextype $>$

$\langle x s:$ complexType name="PIVCardTopographyType" >

$<x s$ : sequence $>$

<xs:element name="EmployeeAfiliation" type="xs:string" minoccurs="0" maxOccurs="1"/>

<xs:element name="AgencyDepartmentorOrganization" type="xs:string" minOccurs="0" maxOccurs="1"/>

<xs:element name="CardexpirationDate" type="xs:date" minoccurs="0" maxOccurs="1"/>

<xs:element name="AffiliationColorCode" minOccurs="0" maxOccurs="1">

<xs:simpleType>

<xs:restriction base="xs:string">

<xs:enumeration value="B"/>

$<x$ : enumeration value="G"/>

$<x$ : enumeration value="W"/>

$</ x s$ : simpletype

$</ x s$ :element $>$

<xs:element name="AgencyCardSerialNumber" type="xs:string" minOccurs="0" maxOccurs="1"/>

$<x s$ :element name="IssuerIdentificationNumber" type="xs:string" minoccurs="0" maxOccurs $=" 1 " />$

$<x s$ :element name="Signature" type="xs:base64Binary" minOccurs="0" maxoccurs="1"/>

<xs:element name="AgencySpecificText4F" type="xs:string" minOccurs="0" maxOccurs="1"/>

$<x s:$ lement name="Rank" type="xs:string" minoccurs="0" maxOccurs="1" / >

$<x s:$ lement name="PDFBarCode" type="xs:base64Binary" minOccurs="0" maxOccurs="1"/>

$<x s: e l e m e n t$ name="Header" type="xs:string" minOccurs="0" maxOccurs="1"/>

$<x s:$ element name="Agencyseal" type="xs:base64Binary" minOccurs="0" maxOccurs="1"/>

<xs:element name="Footer" type="xs:string" minOccurs="0" maxOccurs="1"/>

$<x s:$ element name="PhotoBorder" type="xs:base64Binary" minoccurs="0" maxoccurs="1"/>

$<x s:$ lement name="AgencyspecificData" type="xs:string" minOccurs="0" maxOccurs="1"/>

$<x S$ :element name="OrganizationalAffiliationAbbreviation" type="xs:string" minOccurs="0" maxOccurs="1"/>

$<x s: e l e m e n t$ name="RidgingorNotchedTactileMarker" minoccurs="0" maxOccurs="1"> <xs:simpleType>

<xs:restriction base="xs:string">

$<x$ : enumeration value="Ridging"/>

<xs:enumeration value="Notched Corner" / >

$</ x s$ :restriction>

$</ x$ : simpleType $>$

$\langle/ x s:$ element $>$

$<x s:$ lement name="LaserEngraving" type="xs:boolean" minoccurs="0" maxOccurs="1"/>

$<x s:$ element name="ReturnAddress" type="xs:string" minoccurs="0" maxOccurs="1"/> 
is the property of the U.S. Government. Counterfeiting, altering, or misusing violates Section 499 , Title 18 of the U.S. Code."/> $<x s:$ lement name="AgencySpecificText9B" type="xs:string" minOccurs="0" maxOccurs="1"/>

<xs:element name="AgencySpecificText10B" type="xs:string" minOccurs="0" maxOccurs="1"/>

$<x s$ :element name="CardFrontImage" type="xs:base64Binary" minOccurs="0" maxOccurs="1"/> $</ x$ : sequence

$</ x s$ : complextype $>$

$</ x s:$ schema $>$ 


\section{Appendix B-XML Sample PIVChainOfTrust Record}

$<$ ?xml version="1.0" encoding="utf-8"?>

<nist:PIVChainofTrust xmlns:nist="http://csrc.nist.gov/ns/piv/chain-of-trust/1.0">

$<$ nist:EnrollmentRecord $>$

$<$ nist: Personalinformation $>$

<nist:PrimaryIdentifier >Doe</nist:PrimaryIdentifier $>$

$<$ nist: SecondaryIdentifier $>$ Jane $</$ nist: SecondaryIdentifier $>$

$<$ nist:PrintedName $>$ Jane Doe</nist:PrintedName $>$

$<$ nist: IsUSCitizen $>$ true $</$ nist: IsUSCitizen>

$<$ nist:CitizenshipCountryCode $>$ US $</$ nist:CitizenshipCountryCode $>$

$<$ nist:CardUUID>bb3618a0-4d26-11e5-885d-feff819cdc9f $</$ nist:CardUUID $>$

<nist: CHUID>538206493019D4F81OD9011560100202858360DRB4BC32D14F8184388E13FC3418303030383030303830303030303830303538323031313B333 B343E82068D30820689060929864886F7BD8187829082SFR308205F68201033189300786052BBEB30219388906086386488165030601908284233082841F3882038890 $03020102020219 \mathrm{E} 13000068929864886 \mathrm{~F} 7 \mathrm{BDO} 101050500305031033889060355848613025553311838168603550489130 \mathrm{~F} 552 \mathrm{E} 532 \mathrm{E} 28476 \mathrm{~F} 7665726 \mathrm{E} 6 \mathrm{D} 656 \mathrm{~F} 74318038$ $09860355048 \mathrm{~B} 1303446 \mathrm{~F} 44318 \mathrm{C} 380906035504 \mathrm{OB} 138358484931173815868355040313 \mathrm{OE}$ 44 4F4 4204R4 954432843412D $3133381 \mathrm{E} 17 \mathrm{BD} 3 \mathrm{~B} 373 \mathrm{~B} 31323231393238343459$ 17BD31323031323131393238343459306231BB380986835504861382555331183816860355848913BF552E532E23476F7665726E6D656E74318C388906835504881383 17BD31323031323131393238343459306231BB380986835504861382555331183816860355848913BF552E532E234 76F766572 6E 6D656E74318C388906835504881383 446F4 4313C338906B35584BB13B3534B4 9311D381306035584331314446F44284361726420497373756572205438303738819F300Do6892 9864886F780010101050003 818Do830818902818180D4C2B97DD4F37C1RBD573094286B2058DE9B2 667DDE1924981140673DB3BC8ED285663E85634 75ECC3EE110080902 8DDGE88BFBSR9B8964CBB D982D69050203010801f138201E8308201E4301F0603551D23B41830168014DB88CB96584EFOCD4D107E994EZB4FC173D37917301DO603551DBEB416B414773988D289 E69D889D4EIC7F28C99DS91297697938138683551D2584BC3889060868864801658386873BBE8683551DBFB1B1FFB4848302860038160683551D28840F3BBD38038609 61073831D4383898399037863568747478392F2563726C2E6764732E65697425646973612E6D696C2567657463726C3E44 $\begin{array}{ll} & \end{array}$ 30494954432523043412031332532636 F7525336450484 92532636F7525336444 6F442532636F253364552E532E2532304 76F766572 6E 6D656E742532 636325336455 533F63657274696669636174657265766F636174696F6E6C6973743B62696E61727933818206082886810505873101@476307438420688230601058507388286366874 7478392F2F63726€2E6764732E6E69742E646973612E6D696C2F6765747369676E3F444F442532304f149544325323843412D3133382E8608230681850587308186226 8747470392F2F6F6373782E6E736E382E72 6376732E6E69742E646973612E6D696CBBBD868929864886F7BD818185850883818188B631481DF3DD550297829919FB464 R75063387378203BF3E7DFCICSSERD91F104E562893996CRFD8613EC3B9915929045293710D3B4CDQBB4DFBS4 780F867061918433922259CEOE37BR944504145C258E5 837089942418 CE 382215028nCB3EC4BFF26B372D5F340754DE6787CR3953SF509FE854CZBC9CBF881426917078590318201B1308201900201013062305C31083809060 35504B6130255533118381686035504891305552E532E284767766572656D656E7431003889860355048B1303446544318C30090603550403130350434931173815868 (1) 431168414EDDBB7R710158C6689F66828D73421FCSZB4 60843078068860864881658386853164306231BB380906835584861302555331183316868355843913BF552E5 32E20476F7 665726E6D656E74310C3BBRB6035584BBI 383446F44310€308906035504BB1303504349311D301806835504831314446F442043617264284973737565722 05430383738OB868929864886F7BDo1010584818980162C29697DF641EE7E141C2BC2 9B41689SF96FB77R7 9959E69C4 438DFDC7BBE9128E8D05BDDSRFF8194RB3C4E61 37EB9354B8F3773DC142F7F57E 9E1E24 4BCBFRR3E 94 9D972 4B1BFCR4 9F9E1C2B1CB821B3BD1 f1ECD 9RF636SCD2BE 95CF5C64808879D 67942CF4B18624F4 763F9BBD 713 Do4CDD899FE480629F4235BCEB3884FEBB $</$ nist: CHUID>

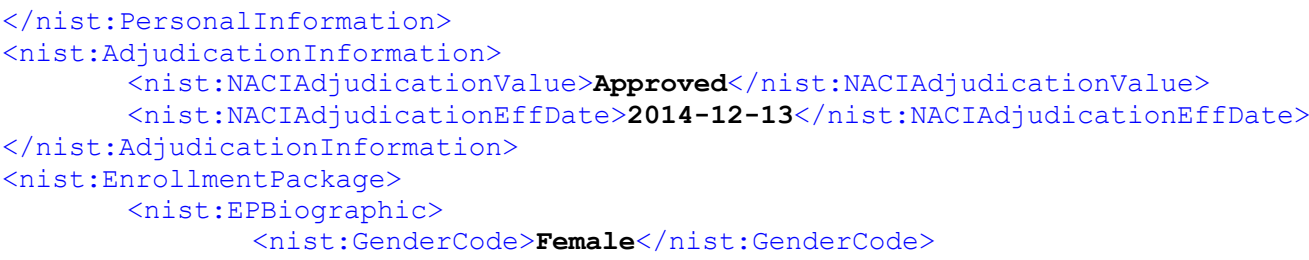




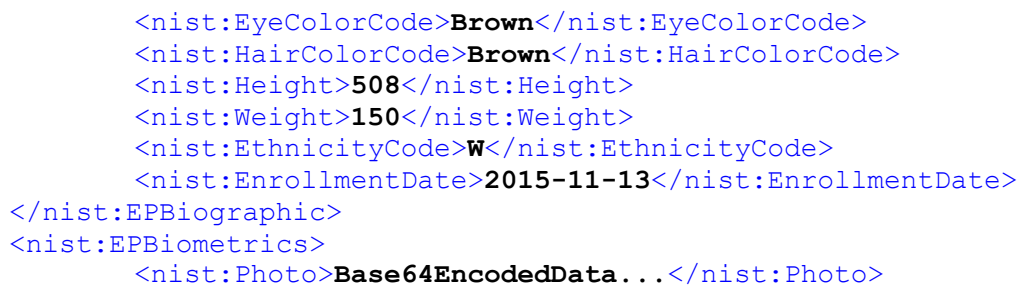

<nist: FingerprintsoffCardComparisonRepresentation>Base64EncodedData. . </nist: FingerprintsoffCardComparisonRepresentation>

$<$ nist: FingerprintonCardComparisonRepresentation>Base64EncodedData . . </nist: FingerprintonCardComparisonRepresentation>

$<$ nist: IrisRepresentation>Base64EncodedData...</nist:RepresentationIris $>$

$$
\begin{aligned}
& </ \text { nist:EPBiometrics }> \\
& <\text { nist:EPDocument> }
\end{aligned}
$$

$<$ nist: DocumentTypeIdentifier>Primary identity source</nist:DocumentTypeIdentifier>

<nist:DocumentTypeCode>Driver's license or an ID card issued by a state or possession of the United

States provided it contains a photograph $</$ nist: DocumentTypeCode $>$

$<$ nist: Document Number $>\mathbf{T 1 2 3 4 5 6 7 8}</$ nist: DocumentNumber $>$

$<$ nist: SequenceNumber $>00001</$ nist: SequenceNumber $>$

$<$ nist: CountryCode $>$ USA $</$ nist: CountryCode $>$

$<$ nist: StateCode $>$ VA $</$ nist: StateCode $>$

$<$ nist:ExpiryDate $>\mathbf{2 0 1 8 - 1 2 - 1 8 < / n i s t : E x p i r y D a t e > ~}$

$<$ nist: Document ImageFront $>$ Base64EncodedData. . </nist: Document Image Front $>$

$<$ nist: Document ImageBack $>$ Base64EncodedData...</nist: DocumentImageBack $>$

<nist: CollectionDate>2012-12-13</nist:CollectionDate>

$</$ nist:EPDocument $>$

$<$ nist:EPDocument>

<nist:DocumentTypeIdentifier>Secondary identity source</nist:DocumentTypeIdentifier>

<nist:DocumentTypeCode>U.S. Social Security Card issued by the Social Security

Administration $</$ nist: DocumentTypeCode $>$

$<$ nist: SequenceNumber $>\mathbf{1 2 3 - 1 2 - 1 2 3 4 < / n i s t : ~ S e q u e n c e N u m b e r > ~}$

$<$ nist: CountryCode $>$ USA $</$ nist: CountryCode $>$

$<$ nist: Document Image Front $>$ Base 64EncodedData...</nist: Document Image Front $>$

<nist: Document ImageBack>Base64EncodedData...</nist: DocumentImageBack>

$<$ nist: CollectionDate $>\mathbf{2 0 1 2 - 1 2 - 1 3 < / n i s t : C o l l e c t i o n D a t e ~}>$

$</$ nist:EPDocument $>$

$</$ nist: EnrollmentPackage $>$

$<$ nist:PIVCardTopography >

<nist:EmployeeAfiliation>Employee</nist:EmployeeAffiliation>

<nist:AgencyDepartmentororganization $>$ DHS $</$ nist: AgencyDepartmentororganization>

$<$ nist: CardExpirationDate $>\mathbf{2 0 1 6 - 1 2 - 1 3 < / n i s t : C a r d E x p i r a t i o n D a t e ~}>$

$<$ nist:AffiliationColorCode $>\mathbf{W}</$ nist:AffiliationColorCode $>$

$<$ nist:AgencyCardSerialNumber $>\mathbf{9 8 7 6 5 4 3 2 1 < / n i s t : A g e n c y C a r d S e r i a l N u m b e r ~}>$

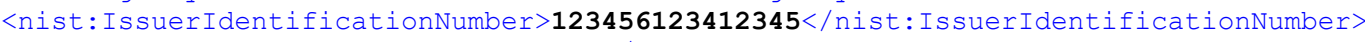

<nist:Signature>Base64EncodedData...</nist:Signature> 
<nist:AgencySpecificText4F>Zone 2 Approved</nist:AgencySpecificText4F>

$<$ nist: $\operatorname{Rank}>$ SES $</$ nist:Rank>

$<$ nist:PDFBarCode>Base64EncodedData...</nist: PDFBarCode>

$<$ nist:Header $>$ United States Government</nist:Header $>$

$<$ nist:Agencyseal>Base64EncodedData...</nist:Agencyseal>

$<$ nist:Footer $>$ Federal Emergency Response Official</nist:Footer $>$

$<$ nist: PhotoBorder>Base64EncodedData...</nist: PhotoBorder $>$

<nist:AgencySpecificData>str1234</nist:AgencySpecificData>

$<$ nist:OrganizationalAffiliationAbbreviation>DHS </nist:OrganizationalAffiliationAbbreviation>

<nist:RidgingOrNotchedTactileMarker>Ridging</nist:RidgingOrNotchedTactileMarker>

$<$ nist: LaserEngraving $>$ true $</$ nist: LaserEngraving $>$

$20528</$ nist : ReturnAddress $>$

nist:ReturnAddress>Return to: Washington Navy Yard, Building 410, 245 Murray Lane SW Washington, DC

ist: AdditionalLanguageForEmergencyResponseofficials>Priority

uageForEmergencyResponseofficials>

<nist:StandardSection499Title18>This credential is the property of the U.S. Government. Counterfeiting,

altering, or misusing violates Section 499 , Title 18 of the U.S. Code.</nist.StandardSection499Title18>

$</$ nist:PIVCardTopography>

$</$ nist:EnrollmentRecord $>$

<ds:Signature xmlns:ds="http://www.w3.org/2000/09/xmldsig\#">

$<$ ds:SignedInfo>

<ds:CanonicalizationMethod Algorithm="http://www.w3.org/2001/10/xml-exc-c14n\#" />

<ds:SignatureMethod Algorithm="http://www.w3.org/2001/04/xmldsig-more\#rsa-sha256" />

$<\mathrm{ds}:$ Reference URI="\# 65c217dd-6589-4830-a277-2083fe4a6183">

$<$ ds:Transforms $>$

<ds:Transform Algorithm="http://www.w3.org/2000/09/xmldsig\#enveloped-signature" />

$<$ ds:Transform Algorithm="http://www.w3.org/2001/10/xml-exc-c14n\#" /

$</$ ds: Transforms $>$

<ds:DigestMethod Algorithm="http://www.w3.org/2001/04/xmlenc\#sha256" />

$</$ ds: Reference>

$<$ ds: DigestValue>BaCypVvGnmkJvmHImOfavpbrq1f4bL9HasRWGmm7HW0 $=</$ ds: DigestValue $>$

$</$ ds: SignedInfo>

dmbTWZKUYFquHclxtaGOoPYt6Y/J7aFSwj5kfaWIQRG6StlVUk/2OB17i IDuvRKOYSjwRTpGOXFikjh+iXuA1M+fRKpdTTHPQx/jyLuZDUhcKLWmK/4Sf1DWC39pRLu Rue5ZSA6zMut+CFEO/x75BQVI2+qwBxCBGLkWV7Bw00JVkYTAV9ILAfP4 PNQopnvYA25XAn7XjLX+a/0cC5/19HB8G1TCKbEmTIBok30W/j4YaofUhz+zp0Q7YZMMAXZDW3P3B S2Jy6C90xnihvqBhrQaSCI+acQ1EvLPq8843IUMjSu6Fxr0NOeBBx9sqa837eMlUzz2UVoo0a83q2CHww==</ds: SignatureValue $>$

$<$ KeyInfo xmlns="http://www.w3.org/2000/09/xmldsig\#">

$<$ X509Data $>$

<X509Certificate>MIIFPzCCBCegAwIBAgIHKySIQq+1ZDANBgkqhkiG9w0BAQUFADCByjELMAkGA1UEBhMCVVMxEDAOBgNVBAgTB0FyaXpvbmExEzARBgNVBACTC1 Njb3R0c2RhbGUxGjAYBgNVBAOTEUdvRGFkZHkuY29tLCBJbmMuMTMwMOYDVOOLEypodHRwOi 8vY2VydGlmaWNhdGVzLmdvZGFkZHkuY29tL3Jl cG9 zaXRvcnkxMDAuBgNVBAMT JOdvIERhZGR5IFN1Y3VyZSBDZXJ0aWZPY2F0aW9uIEF1dGhvcml0eTERMA8GA1UEBRMIMDc5Nj kyODcwHhcNMTQwODE5MTg1NjAXWh CNMTUwODE5MTg1NjAXWjA7MSEwHwYDVO

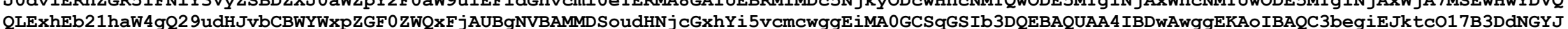

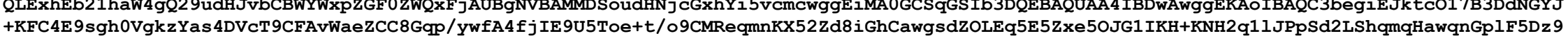

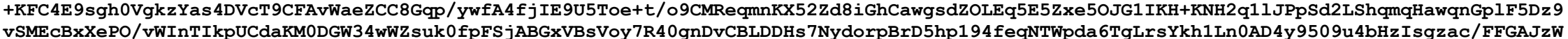

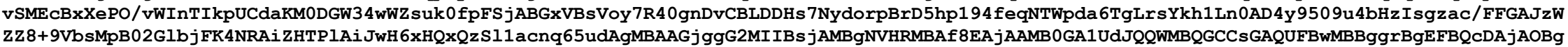
NVHQ8BAf8EBAMCBaAwNAYDVR0fBC0wKzApoCegJYYjaHR0cDovL2NybC5nb2RhZGR5ImNvbS9nZHMxLTExMi5j cmwwUwYDVR0gBEwwSjBIBg tghkgBhv1 tAQcXATA5MDcGCCsG 
AQUFBzIBFi todHRwOi 8vY2VydGlmaWNhdGVzLmdvZGFkZHkuY29tL3JlcG9 zaXRvcnkvMIGABggrBgEFBQcBAQROMH IwJAYIKwYBBQUHMAGGGGh0dHA6Ly9vY3NwLmdvZGFkZH kuY29tLzBKBggrBgEFBOCwAOY+aHR0 cD ovL2Nl cnRpZml jYXRl cy5nb2RhZGR5LmNvbS 9yZXBvc21 Ob3J5L2dkX2ludGVybWVkaWF0 ZS5 jcnOwHwYDVR0 jBBgwFoAU/axhMpNs

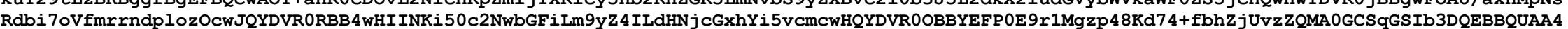
IBAQAx1/u3Nb8mG5Bm4tWV+j+NW2PqWvLVL2qTafk+fNw1I 4 6Rj xmb4+QR2 xZbYytO0z PPa9rszVOy 43SA2+sOD5OAWoUv+P710X87d2 6Nzi/gAJCgfbLj6YMaeOKfut5KIVih h2x/CJbV12w3Gn0Jf6TUYi/6HayKrDVAw1MFNzTS0oFCWOZj 9I1KIyhe99+s+h5rIVjT75ETyPn3jfjmiWr4sXwswjdGhuxOrgq0 tonKI 6NdzQnC1OAgvFCAiMzJT+RmN8Ltat SWESv5YBND8PYJ/1rD2nGVI27S/LkAMeWCQxGf+9wFtzuqzYtbX1nxv5ksvimzJ+cOaa/BcAYkGC10</X509Certificate>

$$
</ \text { X509Data }
$$

$$
\begin{gathered}
</ \text { KeyInf } \\
</ \text { ds: Signature }
\end{gathered}
$$

$</$ nist:PIVChainofTrust> 


\section{Appendix C-References}

[EBTS]

[FIPS140]

[FIPS201]

[HSPD12]

[ISO3166]

$[\mathrm{M}-07-16]$

[RFC2119]

[SP800-73-4]

[SP800-76]
Criminal Justice Information Services. Electronic Biometric Transmission Specification (EBTS) 10.0, July 2, 2013, https://www.fbibiospecs.cjis.gov/Document/Get?fileName=Master \%20EBTS\%20v10\%20-\%20FINAL\%2020130702.pdf [accessed $5 / 10 / 16]$.

National Institute of Standards and Technology, Security Requirements for Cryptographic Modules, Federal Information Processing Standards (FIPS) Publication 140-2, May 2001 (including change notices through 12/3/02), $61 \mathrm{pp}$. http://csrc.nist.gov/publications/fips/fips140-2/fips1402.pdf [accessed 5/10/16].

National Institute of Standards and Technology, Personal Identity Verification (PIV) of Federal Employees and Contractors, Federal Information Processing Standards (FIPS) Publication 201-2, August 2013, 87pp. http://dx.doi.org/10.6028/NIST.FIPS.201-2.

Homeland Security Presidential Directive-12, Policies for a Common Identification Standard for Federal Employees and Contractors, August 27, 2004. http://www.dhs.gov/homelandsecurity-presidential-directive-12 [accessed 5/10/16].

International Organization for Standardization, Codes for the representation of names of countries and their subdivisions, https://www.iso.org/obp/ui/\#search [accessed 5/10/16].

Office of Management and Budget (OMB), Safeguarding Against and Responding to the Breach of Personally Identifiable Information, https://www.whitehouse.gov/sites/default/files/omb/memoranda/fy2 007/m07-16.pdf [accessed 5/11/16]

S. Bradner, Key words for use in RFCs to Indicate Requirement Levels, Internet Engineering Task Force (IETF) Network Working Group Request for Comments (RFC) 2119, March 1997 https://dx.doi.org/10.17487/rfc2119.

National Institute of Standards and Technology, Interfaces for Personal Identity Verification - Part 1: PIV Card Application Namespace, Data Model and Representation, NIST Special Publication (SP) 800-73-4, May 2015 (updated 2/8/16), http://dx.doi.org/10.6028/NIST.SP.800-73-4.

National Institute of Standards and Technology, Biometric 
Specifications for Personal Identity Verification, NIST Special Publication (SP) 800-76-2, July 2013,

http://dx.doi.org/10.6028/NIST.SP.800-76-2.

[SP800-79-2] National Institute of Standards and Technology, Guidelines for the Authorization of Personal Identity Verification Card Issuers (PCI) and Derived PIV Credential Issuers (DPCI) , NIST Special Publication (SP) 800-79-2, July 2015, http://dx.doi.org/10.6028/NIST.SP.800-79-2.

[USPS-Appendix United States Postal Service, Appendix B: Two-Letter State and B] Possession Abbreviations, in Publication 28, Postal Addressing Standards, PSN 7610-03-000-3688, May 2015, http://pe.usps.gov/text/pub28/28apb.htm [accessed 5/10/16].

[XMLEnc] World Wide Web Consortium, XML Encryption Syntax and Processing Version 1.1, W3C Recommendation 11 April 2013, http://www.w3.org/TR/2013/REC-xmlenc-core1-20130411/ [accessed 5/10/16].

[XMLSchema] World Wide Web Consortium, XML Schema, October 15, 2014, http://www.w3.org/2001/XMLSchema [accessed 5/10/16].

[XMLSig] World Wide Web Consortium, XML Signature Syntax and Processing (Second Edition), W3C Recommendation 10 June 2008, http://www.w3.org/TR/xmldsig-core/\#sec-Versions [accessed $5 / 10 / 16]$. 


\section{Appendix D-Glossary of Terms}

Biometric

Cardholder

Chain-of-trust

FASC-N

Issuer

Off-Card

On-Card
The stored electronic information pertaining to a biometric. This information can be in terms of compressed pixels or in terms of some characteristic (e.g., patterns).

An individual possessing an issued PIV Card.

The chain-of-trust is a sequence of related enrollment data sets that is created and maintained by PIV Card issuers

Federal Agency Smart Credential Number (FASC-N): As required by [FIPS201], one of the primary identifiers on the PIV Card. The FASC-N is a fixed length (25-byte) data object, specified in [SP80073-4], and included in several data objects on a PIV Card.

An agency or service provider that issues PIV credentials in accordance with [FIPS201] and NIST [SP 800-79-2].

A cardholder's biometric reference data stored on the PIV Card that is matched against the cardholder's life-scan biometrics Off-Card during authentication.

A cardholder's biometric reference data stored on the PIV Card that is matched against the cardholder's life-scan biometrics On-Card during authentication. 


\section{Appendix E-Acronyms}

\begin{tabular}{|c|c|}
\hline CHUID & Card Holder Unique Identifier \\
\hline $\mathrm{DN}$ & Distinguished Name \\
\hline EVSSL & Extended Validation Secure Sockets Layer \\
\hline FASC-N & Federal Agency Smart Credential Number \\
\hline FBCA & Federal Bridge Certificate Authority \\
\hline FIPS & Federal Information Processing Standards \\
\hline FISMA & Federal Information Security Modernization Act \\
\hline FTPS & File Transfer Protocol Secure \\
\hline HTTP & Hypertext Transfer Protocol \\
\hline HTTPS & Hypertext Transfer Protocol Secure \\
\hline HSPD & Homeland Security Presidential Directive \\
\hline ITL & Information Technology Laboratory \\
\hline NAC & National Agency Check \\
\hline NACI & National Agency Check with Inquiries \\
\hline NIST & National Institute of Standards and Technology \\
\hline OCC & On-Card Comparison \\
\hline $\mathrm{OMB}$ & Office of Management and Budget \\
\hline PDF & Portable Data File \\
\hline PII & Personally Identifiable Information \\
\hline PIV & Personal Identity Verification \\
\hline RFC & Request for Comment \\
\hline S/MIME & Secure/Multipurpose Internet Mail Extensions \\
\hline USPS & United States Postal Service \\
\hline UUID & Universally Unique Identifier \\
\hline
\end{tabular}


W3C

XML
World Wide Web Consortium

Extensible Markup Language 